FERMILAB-Pub-87/174-E

[E-741/CDF]

\title{
The CDF Central and Endwall Hadron Calorimeter*
}

S. Bertolucci, M. Cordelli, B. Esposito, M. Curatolo, P. Giromini, S. Miscetti, and A. Sansoni Laboratori Nazionali dell' INF.N., Frascati, Italy

G. Apollinari, F. Bedeschi, S. Belforte, G. Bellettini, N. Bonavita, F. Cervelli, G. Chiarelli, R. Del Fabbro, M. Dell'Orso, E. Focardi, P. Giannetti, A. Menzione, R. Paoletti, G. Punzi, L. Ristori, A. Scribano, P. Sestini, A. Stefanini, G. Tonelli, and F. Zetti IN.F.N. and University of Pisa, Pisa, Italy

V. E. Bames, A. Di Virgilio, A. F. Garfinkel, S. E. Kuhlmann, and A. T. Laasanen Department of Physics, Purdue Universiry, West Lafayette, Indiana 47907 USA

H. Jensen and H. Kautzky

Fermi National Accelerator Laboratory, Batavia, Illinois 60510 USA

July-August 1987

*Submitted to Nucl. Instrm. Methods A 


\section{THE CDF CENTRAL AND ENDWALL HADRON CALORIMETER}

S. Bertolucci, M. Cordelli, B. Esposito, M. Curatolo, P. Giromini, S. Miscetti and A. Sansoni

Laboratori Nazionali dell'I.N.F.N., Frascati, Italy

G. Apollinari, F. Bedeschi, S. Belforte, G. Bellettini, N. Bonavita, F. Cervelli, G. Chiarelli, R. Del Fabbro, M. Dell'Orso, E. Focardi,

P. Giannetti, A. Menzione, R. Paoletti, G. Punzi, L. Ristori, A. Scribano,

P. Sestini, A. Stefanini, G. Tonelli and F. Zetti

INFN and University of Pisa, Pisa, Italy

V. E. Barnes, A. Di Virgilio, A. F. Garfinkel, S. E. Kuhlmann and A. T. Laasanen

Department of Physics, Purdue University, W. Lafayette, IN, 47907 USA

$H$. Jensen and $H$. Kautzky

Fermi National Accelerator Laboratory, Batavia, IL, 60510 USA

ABSTRACT

The CDF Central and Endwall hadron calorimeter covers the polar region between 30 and 150 degrees and a full $2 \pi$ in azimuth.

It consists of 48 steel-scintillator Central Modules with $2.5 \mathrm{~cm}$ sampling and 48 steel-scintillator Endwall modules with $5.0 \mathrm{~cm}$ sampling. A general description of the detector is given. Calibration techniques and performance are discussed. Some results of the test beam studies are shown. 


\section{Detector geometry}

The large angle CDF hadron calorimeter has cylindrical symmetry and covers polar angles between 30 and 150 degrees. It consists of 48 steel-scintillator Central calorimeters with $2.5 \mathrm{~cm}$ sampling and 48 steel-scintillator Endwall calorimeters with $5.0 \mathrm{~cm}$ sampling.

Each calorimeter module is divided into projective towers, each covering approximately 0.1 unit in pseudo-rapidity and 15 degrees in azimuthal angle, matching those of the Electromagnetic calorimeter, which is in front of it. This segmentation is fine enough that quark and gluon jets will normally spread over more than one tower.

For each 15 degree azimuthal slice there are 24 towers in all, of which 12 are totally in the Central calorimeter, 6 totally in the Endwall calorimeter, and 6 are shared.

The Central modules, covering polar angles between 45 and 135 degrees, are 32 layers deep and weigh about $12,000 \mathrm{~kg}$ each ( Fig. 1 ). They are stacked into four free standing " $\mathrm{C}$ " shaped arches which can be rolled into and out of the detector.

The Endwall modules (Fig. 2) cover polar angles from 30 to 45 and from 135 to 150 degrees and weight about $7,000 \mathrm{~kg}$ each. They plug into cavities in the CDF magnet yoke and serve as part of the flux return path. Fig. 3 shows one quadrant of the large angle electromagnetic and hadron calorimeters segmented into 12 polar towers.

Tables 1 and 2 summarize various parameters of the large angle hadron calorimetry.

\section{Light collection system, photomultipliers and front-end electronics}

The scintillating plastic is PMMA doped with $8 \%$ Naphthalene, $1 \%$ Butyl-PBD and $0.01 \%$ POPOP [1]. The scintillators, $1 \mathrm{~cm}$ thick, are shaped to give a tower geometry and have average dimensions of $1 \times 35 \times 70 \mathrm{~cm}^{3}$.

Fig. 4 shows how the light is collected by wavelength shifter (WLS) strips which lie against the long sides of the scintillator sheets. These 
$0.5 \times 1.0 \mathrm{~cm}^{2}$ UVA PMMA strips [1] are doped with laser dye \#481 [2], which has an emission peak at about $490 \mathrm{~nm}$. The WLS strips butt up against clear UVA light guides [3] of the same cross-section which are collected to form two square arrays at the azimuthally opposite sides of the tower. Each square array is glued to a transition piece of PMMA doped with the laser dye \#481 which matches the square cross-section to the circular photocathode of a photomultiplier tube. The light from each tower is thus collected by two photomultiplier tubes on opposite sides in azimuth.

Phototubes for both the Central and Endwall were chosen to satisfy the following requirements:

a) quantum efficiency better than $10 \%$ for light from laser dye \#481;

b) linearity up to peak currents of $40 \mathrm{~mA}$ at a gain of $10^{6}$ and voltages of about $1500 \mathrm{~V}$;

c) high gain stability ( $<2 \%$ variation) for uninterrupted operation and for anode currents $l_{a}$ between .5 and $15 \mu \mathrm{A}$ (hypothesized current changes between Collider operation mode and calibration periods with no beam).

Accordingly we chose the 12 stage THORN-EMI 9954 phototube for the Central calorimeter and the 10 stage THORN-EMI 9902 tube for the Endwall [4].

Because of a later change of the ADC full scale, those photomultipliers are run at a gain of $10^{5}$ with $l_{a}$ ranging from some tens to a few hundreds nano-Amperes. In this untested range, the 9954 photomultipliers exhibit gain changes up to $20 \%$ as a function of $I_{a}$, (Fig. 5). To minimize this instability, these photomultipliers are illuminated, between bunch crossings, with green light, producing an anode current of about $100 \mathrm{nA}$. With this fix and after having replaced many photomultipliers, less than $1 \%$ of all tubes show gain changes bigger than $2 \%$ as a function of $I_{a}$.

Using the Rabbit readout system [5], anode signals are digitized by a 16 bit ADC with a full scale of $750 \mathrm{pC}$. The system is also capable of digitizing the phototube currents. The arrival time of signals from the last dynode of the phototubes is digitized using a 16 bit TDC with a full scale of $3.2 \mu \mathrm{sec}$.

\section{Design constraints}


The design of hadron calorimetry for an experiment like CDF obviously stresses the best possible energy resolution and the best possible uniformity.

The scintillator hadron calorimeter was designed to have a $2.5 \mathrm{~cm}$ sampling, aiming for a percentage energy resolution $\sigma(E) / E=.5 / \sqrt{ } E(\mathrm{Gev})$. Nevertheless financial and installation constraints resulted in a total thickness of $80 \mathrm{~cm}$ of steel at normal incidence $(95 \%$ containment at $50 \mathrm{GeV})$. This degrades the calorimeter resolution at higher energies and makes the muon identification in the chambers behind the calorimeter more difficult. Still, based on prototype results, the 2.5 $\mathrm{cm}$ sampling (32 scintillator layers) was chosen for the Central calorimeter in order to keep a better resolution at lower energies [6], whereas $5.0 \mathrm{~cm}$ was chosen for the Endwall, since, for a given $E_{t}$, the total energy in this calorimeter is on average a factor $\sqrt{ } 2$ bigger than in the Central.

To avoid further deterioration of the calorimeter performance, special attention was paid to response uniformity. For instance, longitudinal (depth) uniformity not only avoids resolution degradation due to spatial fluctuations of the shower, but also implies that different modules have the same ratio between minimum ionizing and showering particles. This is important, because it makes absolute calibration possible without the need for exposing every module to a test beam.

Uniform angular response was achieved through a careful study of the readout scheme, taking into account optical properties of the scintillator and wave length shifter, as well as geometrical constraints.

Fig. 6a shows the response of a scintillator, which has the average dimensions of those present in Central modules, when irradiated by a $\mathrm{Sr}^{90} \beta$ source and with the light collected from the short (azimuthal) side through a wave length shifter. Due to the presence of the four tapered edges, which have to accomodate the EM shower counter light pipes, $x(\theta)$ nonuniformities up to a factor 2 are clearly seen for fixed $y$.

Fig. 6b shows that an untapered scintillator certainly has a better $x(\theta)$ behaviour, but still would have nonuniformities of up to $30 \%$ along the perpendicular $y(\phi)$ direction had it been read out from both sides, 
because of the short attenuation length of the scintillator $(35 \mathrm{~cm})$.

Fig. 7 finally shows the response map of the same scintillator used in Fig. 6a, when read out at $\theta=$ constant (see Fig. 4) through wave length shifter strips (attenuation length $\lambda=150 \mathrm{~cm}$ ). Its response map shows variations which are typically (except close to the edges) less than $10 \%$. This nonuniformity is further smoothed out by alternating the WLS read-out in successive calorimeter layers. In this scheme the ratio of charges collected by the two photomultipliers will still allow a fair $\phi$ resolution, provided the number of photoelectrons/GeV is high enough.

The calorimeters' longitudinal response was made uniform to better than $10 \%$ by a two step procedure.

A first step was performed at the construction time. Since the scintillation light output was found to change monotonically as a function of the scintillator dimensions and therefore of its longitudinal location, a coarse correction was obtained by coupling scintillators to light guides selected according to their $\lambda$. The second step made use of filters at the couplings between the wave length shifters and light guides.

Their insertion reduces the total light output at the phototubes by $20 \%$ on average (see Fig. 8 ). The uniformity of longitudinal sensitivity was determined by measuring the phototube current induced by a $\mathrm{Cs}^{137}$ point $\gamma$ source which was moved longitudinally along the tower (this will be referred to as a "skin source"). Since the $y$ source illuminates more than one scintillator at a time, the effect of the source on a single scintillator was calculated [7] and consistently checked with measurements performed with a $\beta$ source positioned at the center of each scintillator.

\section{Calibration monitoring systems}

The calibration maintenance systems for Central and Endwall calorimeters have been designed to insure monitoring of the phototube gains and to check possible deterioration of scintillators, wave shifters and light guides. The primary system for monitoring the phototube gains uses a pulsed laser whose light is diffused and distributed by optical fibers to the WLS doped coupling blocks in front of all photocathodes. A backup system uses a point-like $\beta$ source that 
can be inserted into the coupling blocks between the light guides and the phototubes. Ageing effects can be detected by comparing the results of the previous systems with those of two other monitoring systems which induce currents by irradiating the calorimeter's scintillator with $\gamma^{\prime}$ s from $\mathrm{Cs}^{137}$ sources. The first one of these automatically moves a point source, at constant speed, over a plane of scintillators at a fixed depth in each module. The second one consists of the longitudinal insertion into any tower of a radioactive line source, which simultaneously irradiates all layers.

\section{4a. The Laser System}

The laser system is shown in Fig. 9. A nitrogen laser [8] provides light pulses with wavelength of $320 \mathrm{~nm}$, at a repetition rate of $15 \mathrm{~Hz}$ and a power output of approximately $0.3 \mathrm{~mJ} / \mathrm{pulse}$. Light pulses are a few nanoseconds long and reasonably constant (within $5 \%$ ) over the period of use.

The laser beam is split into 6 beams which are transported to the calorimeters via 6 optical fibers, 4 for use on the Central calorimeters and 2 on the Endwalls. The intensity of each beam is equalized by diaphragms. A set of neutral density filters on the main laser beam allow linearity checks of the PM's and calibration of the ADC range. The $61000 \mu \mathrm{m}$ thick quartz optical fibres have an attenuation length of $100 \mathrm{~m}$ and are $60 \mathrm{~m}$ long. They terminate in light distribution boxes on the detector, where they illuminate the axis of a scintillator disc (PMMA doped with $0.005 \%$ BBOT) through an air gap. The disks (10 mm high and $70 \mathrm{~mm}$ in diameter) have a surface with a conical depression so shaped that the laterally emitted light will uniformly illuminate the ends of a set of optical fibers which carry the light to the phototubes. These are radially arranged around the disk (Fig. 10) into 13 bundles, each containing fourteen $200 \mu \mathrm{m}$ thick fibers. Twelve of these bundles are sent to a set of calorimeter modules while the thirteenth returns to a monitor system in the counting room. The light sent to each p.m. is equalized to within $10 \%$ by adjusting small threaded cylinders at the fibers' end, which act as limiting apertures. The fibers of the 13th (reference) bundle are sent to 4 photomultipliers contained in a temperature stabilized box, located in the counting room. The gains of the reference phototubes are monitored by illuminating them with light from $\mathrm{Nal}$ crystals [9], doped with $\alpha$ 
emitting $\mathrm{Am}^{241}(\sim 1000$ counts $/ \mathrm{sec})$.

4b. The line source system

The use of an extended radioactive source, which simultaneously illuminates all scintillators of a calorimeter tower, allows one to monitor the response of the complete system of scintillators, wavelength shifters, light pipes, and phototubes. We chose a linear Cs137 y source with a total strength of $3 \mathrm{mCi}$ encapsulated in a flexible $0.5 \mathrm{~mm}$ stainless steel tube, $1.6 \mathrm{~m}$ long. The source can be inserted into two larger diameter stainless steel tubes fixed longitudinally on both sides of each tower as shown in Fig. 2. The tubes have been positioned along the centerline of the tower to within a few millimeters.

\section{4c. The movable point source system}

This system, operating under computer control, is capable of moving individual $1.3 \mathrm{mCi}$ point-like $\gamma$ sources into each module at a fixed longitudinal depth (see Fig. 2). Moving inside a thin stainless steel tube, the source traverses all towers of a module along the scintillator centerlines. When not in use the sources reside in lead blocks mounted outside the modules. The system measures the product of the response of the scintillators at a single level and the gain of the phototubes.

\section{$4 d$. The point $\beta$ source system}

Provision has been made for the manual insertion of point-like $\beta$ sources into the coupling blocks between the light guides and the phototubes.

The insertion of a standard source gives a direct measurement of the phototube gain in situations where the laser system is not available. It was primarily used during the test beam calibration of the Central modules.

5. Test beam results for Central and Endwall modules 
The Fermilab N_West charged particle beam was used for testing and primary calibration of the Central and Endwall calorimeter modules. The modules were mounted on a specially fabricated carriage, which automatically swept them through the beam in both polar and azimuthal directions. Besides the primary calibration at the tower center, some preliminary studies of the calorimeter uniformity were also performed.

\section{5a. Response to muons}

The response of the calorimeter to muons was studied both in the test beam and with cosmic rays. The test beam results will be presented here, while the bulk of the cosmic ray results will be presented elsewhere [10]. Fig. 11 shows a typical muon spectrum of an Endwall tower. Table 3 summarizes the ratios of pion to muon responses at $50 \mathrm{GeV}$ as a function of the tower number. Averaging over the unshared towers of the Central calorimeter, the ratio is about 25 . A similar result holds for the Endwall. This corresponds to an equivalent muon energy of about $2.0 \mathrm{GeV}$. It corresponds to $16 \mathrm{ep} / \mathrm{GeV}$ for the Central and about $8 \mathrm{ep} / \mathrm{GeV}$ for the Endwall, defining $\mathrm{ep} / \mathrm{GeV}$ (number of equivalent particles per $\mathrm{GeV}$ ) as the ratio between the number of scintillator layers in a tower and the energy deposited in the tower by a muon. We estimate that we collect approximately 40 photoelectrons per $\mathrm{GeV}$ in a Central calorimeter tower and half that number in an Endwall.

\section{5b. Linearity response and energy resolution}

Fig. 12 shows the peaks of the energy distributions in the Central calorimeter when exposed to pions in the energy range 10 to $150 \mathrm{GeV}$ hitting the center of the fifth tower. Events were selected requiring the primary pion interaction to occur in the hadron calorimeter. Deviations from linearity, while not visible on this plot, are expected due to leakage at high energy, to reduced calorimeter response at low energy [11] and to poor knowledge of the beam at low energies. Further studies are planned.

Fig. 13 and 14 show the pulse height distributions for $50 \mathrm{GeV}$ pions which interact in Central tower 1 and Endwall tower 10. Fig. 15 shows 
the total (hadron plus electromagnetic calorimeter) pulse height distribution for $50 \mathrm{GeV}$ pions which interact either in the Central tower 1 or in the electromagnetic compartment in front of it. Fig. 16 shows the dependence of percentage energy resolution on beam energy for pions hitting towers 1 and 5 of the Central calorimeter $(2.5 \mathrm{~cm}$ sampling) and tower 10 of the Endwall $(5.0 \mathrm{~cm}$ sampling). These pions were selected to be minimum ionizing particles (m.i.p) in the e.m calorimeter and the resolution is defined by the sigma/peak ratio, including only events within two sigma from the peak. The figure shows that, at low energies, the resolutions of towers 1,5 and 10 , when plotted versus $1 / \sqrt{ }(E)$, lie approximately on straight lines of increasing slopes, according to the increasing sampling thickness. As energy increases the contribution from leakage is predominant: for instance, beyond $50 \mathrm{GeV}$, the energy resolution in tower 5 , which has a factor 1.4 coarser sampling but is a factor 1.4 longer, is better than in tower 1.

\section{5c. Time response}

The time of flight resolution of the sum of the photomultipliers of a Central hadron calorimeter tower, measured with the pion beam and with cosmic rays [12], was found to be $1.5 \mathrm{~ns}$. The time dependence upon the pion position in a tower was also measured and found to be less than $0.5 \mathrm{~ns}$. The time dependence as a function of pulse height was also measured and will be corrected for.

\section{5d. Calorimeter response map}

A refined study of the calorimeter response map, and in particular the study of interface problems, requires exposing two adjacent modules to the test beam. Due to time constraints, only a minor part of this program has been performed and most of the data presented here refer to a single module.

Fig. 17 shows the response to a $50 \mathrm{GeV}$ pion beam, when one module is centered in the $\phi$ direction and swept in the $\theta$ direction. The total response is independent of the $\theta$ location of the shower, only suffering a $10 \%$ dip when the beam is centered in between two adjacent towers. This is due to the poorer light output of WLS compared to that of scintillator. 
With the beam hitting the tower centers, leakage into adjacent towers was found to vary monotonically with polar angle between $6 \%$ at 90 degrees to $12 \%$ at 30 degrees, (Fig. 18), while azimuthal leakage was negligible.

Two stacked modules were swept through a pion beam to study the $\phi$ behaviour of the calorimeter. Fig. 19 shows the response, averaged over the beam size (radius $\sim 2 \mathrm{~cm}$ ), to a $\phi$ scan along the tower $\theta$ centers with $50 \mathrm{GeV}$ pions. While the response is flat when the beam is inside one of the modules, the interface region not only shows a dip, but also shows a huge degradation of the energy resolution, indicating the presence of low (dead areas) and high tails (hot spots). In the well behaved region, a measure of the $\phi$ position of the shower can be extracted from the logarithm of the ratio of the two photomultipliers in a tower, $\ln (L / R)$, because the collected light drops exponentially with the distance between the shower and the photomultipliers.

Fig. 20 shows $\ln (L / R)$ as function of $x$, where $x$ is the distance of the beam from the center of a scintillator located at the shower maximum, yielding an equivalent attenuation length $(\lambda)$ of $164 \mathrm{~cm}$.

The resolution dependence on position and energy is shown in

Fig. 21. At tower center it varies between 3 and $7 \mathrm{~cm}$ for particle energies ranging from 50 to $15 \mathrm{GeV}$, corresponding to an angular resolution of 0.6 to 1.4 degrees respectively.

A more detailed study of the interface between the two modules was performed, selecting the position of the incoming particles with wire chambers put in front of the modules.

Fig. 22 shows the calorimeter response to $50 \mathrm{GeV} e^{-}$impinging on this interface area. Three distinct regions show up. A first one, outside the peaks, corresponds to the "regular" region, where the small energy observed accounts for the leakage coming from the shower in the e.m. compartment; the second, between the peaks, corresponds to a dead area due to $\theta^{-}$showering in the steel skins of the modules; the third one, the "hot spot" region, shows huge pulse heights, up to 2-3 times the expected values, due to Cerenkov radiation produced by showers in the light guide trees. These "hot spots" were found to be fairly linear as a function of the $\theta^{-}$energy (Fig. 23). A similar behaviour, although less dramatic in frequency of occurrence and released energy, was measured for pions impinging on the same area.

Masking the crack in the front of the modules with a radiator, greatly reduces the effect for $e^{-}$and $\gamma^{\prime}$, as shown. in Fig. 23 for 
different radiator thicknesses.

Crack fillers, consisting of about 10 radiation lengths $\left(X_{0}\right)$ of $U^{238}$ and followed by a wire chamber, were installed. They not only eliminate the effect, but also provide a tag for troublesome events and partially recover the energy information in this region. Crack fillers do not cure "hot spots" induced by pions and therefore a method, based on the ratio of the two p.m.'s ( $L$ and $R$ ) in a tower, was developed to tag this class of events. Indeed, for "hot spot" events, this ratio is abnormally high or low, while for "normal" events it is distributed around an energy independent mean value:

$$
\left\langle L R>=e^{-2 \times / \lambda}\right.
$$

(where $x$ is the distance of the shower from the scintillator center and $\lambda$ is the attenuation length at the shower maximum), ranging from $\dot{1} .0$ to 0.6 when moving from the center to the edge of the module. At any given position, the width of the distribution depends on the number of photoelectrons and can be parametrized as:

$$
\sigma(E)=0.04+0.33 / \sqrt{ } E
$$

This formula is used in order to apply an energy independent cut.

Fig. 24 shows the behaviour of $\sigma$ as a function of $1 / \sqrt{ } E$, together with the fitted line.

Fig. 25 shows the scatter plot relating the energies seen by the the two stacked modules, when an electron beam is aimed at the crack.

Using the observed energy on each module to compute the corresponding $\sigma(E)$, each event with $(L / R<(0.6-3 \sigma)$ or $(L / R>1 /(0.6-3 \sigma))$ was rejected. The result of this procedure is shown in Fig. 26, where "hot spots" have disappeared.

\section{Calibration procedure}

The procedure adopted for the absolute calibration made use of the good longitudinal uniformities of the towers, which shortened the turnover time of modules in the test beam and provided an absolute calibration without the exposure of each module to the beam. The Central modules, all of which, however, were test beam calibrated, were used to test this approach.

Initially a couple of modules were exposed to a pion beam and gains were adjusted to yield $2 \mathrm{pC} / \mathrm{Gev}$ on each photomultiplier for 
pions hitting the tower center. At the same gain the mean current $I_{c}$ produced by the "skin source" was measured by averaging the induced currents over all the layers (see Fig. 8). If the towers are longitudinally uniform, then this set of values can be used to fix the gains in the rest of the calorimeter. According to this assumption, the high voltage of each photomultiplier in all the remaining modules was adjusted to get the proper $l_{c}$, after gain conditioning through LED induced dark current (see sect. 2). The currents $I_{S r 90}$ and $I_{C s 137}$, induced by the $\mathrm{Sr}^{90} \beta$ source and by the movable $\mathrm{Cs}^{137}$ source, were also recorded. The modules were then sent to the test beam, where the p.m.'s high voltages were turned on at the nominal values found in the assembly hall. A single calibration point was taken for each tower, recording the charge collected for $50 \mathrm{GeV}$ pions hitting the tower centers. Before and after the calibration run, the values of $\mathrm{I}_{\mathrm{Srgo}}$ and $\mathrm{I}_{\mathrm{Cs} 137}$ were again recorded.

To check the ability of the sources to transfer the calibration from the test beam to the experiment, some modules have been repeatedly calibrated in the beam over a span of several months. If the source system is adequate, one would expect the ratio between the pion peaks of any two calibration runs to equal the ratio of the associated $I_{C_{S 137}}$ measurements; if no deterioration effects are present, this value should also be equal to the ratio of the $I_{S r 90}$ currents. Fig 27 shows the distribution of the percentage difference between corresponding pion peaks of the recalibrated modules; scaled by the $I_{\text {Cs137 }}$ ratios: the standard deviation of the distribution is about $1 \%$. When all the modules were assembled in the experiment, the role of the now impractical $\mathrm{Sr}^{90}$ source was taken over by the laser system.

Once all the 50 Central modules were tested, their degree of similarity was checked by correlating, again through $I_{S r 90}$ and $I_{C s 137}$, the observed pion pulse heights to the average currents $\mathrm{I}_{c}$. Fig. 28 shows that the modules can be calibrated at a level of $3-4 \%$ with the source systems only.

Endwall modules have been calibrated relying on this result. At the test beam the phototube high voltages on two Endwall modules were adjusted to provide $100 \mathrm{pC}$ for $50 \mathrm{GeV}$ hadronic energy deposition. At the same voltages the current induced by $\mathrm{Cs}^{137}$ line source, 
longitudinally irradiating the whole tower (see sect. 4b), was measured for each phototube. On both modules the currents of corresponding towers proved to be equal at a $2 \%$ level for different running conditions. These calibration currents (different for each tower of a module) were set in all the other calorimeter modules by adjusting the high voltage on the phototubes. This procedure of manually using the line $\mathrm{Cs}_{137}$ source required 15 minutes per module. Immediately after the HV setting of each module, the current induced by the movable point source (see sect. 4c) and the laser pulse heights were recorded to provide a reference for each photomultiplier.

\section{Magnetic field effects}

Since the Endwall calorimeter serves as part of the magnetic field return yoke, its scintillators and its phototubes are influenced by the magnetic fringe field. Independent current measurements with the movable point radioactive source and with the laser system allow one to separate magnetic field effects on phototubes from those on scintillators. The percentage displacements of these currents from the initial status of no field have been measured for Endwall phototubes with the super conducting solenoid powered to provide $15 \mathrm{kG}$. As shown in figs 29 and 30 the movable source currents increase on average by $5 \%$, while the mean of the laser induced pulse heights is affected by a smaller amount (about 1\%). Fig. 31 shows the difference, tube by tube, of the quantities plotted in figures 29 and 30 . The prevailing effect, causing the $5 \%$ displacement of the mean value of the distribution, is due to a growth of the scintillator light output in the presence of the magnetic field: this effect is in more detail described in ref. [13].

\section{Radiation damage}

Scintillator samples were uniformly irradiated to measure radiation induced damage.

The observed dose was monitored with TLD's which had been previously calibrated against a reference $\mathrm{Co}^{60}$ source. Attenuation length $\lambda$ was measured for different dose values and the results are shown in Fig. 32.

$\lambda$ varied linearly with the absorbed dose $R$ and decreased by a 
factor of about 4 between 0 and $15 \mathrm{Krad}$. $\mathrm{I}_{0}$, i.e. the light intensity at zero distance, does not change, thus excluding major molecular damage. 


\section{Acknowledgements}

Research supported in part by the U.S. Department of Energy under contracts DE-AC02-76ER01428 and DE-AC02-76CH0300O.

We would like to acknowledge the University of Pennsylvania group (D. Bauer, D. Connor, J. Cooper, S. Hahn, M. Miller, H. H. Williams) for the design, set-up and debugging of the $\mathrm{Cs}^{137}$ point source systems used with the central calorimeter.

We wish to acknowledge T. Devlin, U. Joshi and K. Kazlauskis of Rutgers University for the testing of the Endwalls photomultiplier tubes.

The NW Wedge Group [14] is thanked for having made beam calibration possible. J. Cooper, as leader of the NW Wedge Group, and D. Theriot, as organizer of the detector installation, are particularly acknowledged for organizing smooth operations.

We thank J. Elias for his contributions throughout all the stages from design to installation.

The skills and dedication of D. Allen, M. Biagioli, R. Bonini, W. Burley, J. Catalanello, J. Craig, C. Crow, M. Del Colletto, B. Dulach, E. Dunk, G. Favati, M. Favati, M. Hrycyk, L. lannotti, R. Krull, P. Locchi, H. Martin, D. Pistoni, R. Ruberti, A. Rutili, M. Santoni, C. Schanke, G. Sensolini and $D$. Tinsley have contributed greatly to the actual construction of the Calorimeter. 


\section{Figure captions}

1: Central calorimeter module. A light guide is sketched: scintillators in successive layers are read from opposite $\Theta$-sides. For each tower light is collected by two symmetrically positioned guides.

2: Endwall calorimeter module. Module side faces are equipped with steel tubes positioned on the center lines of the towers and used for insertion of linear $\gamma$ sources (sect.4b). The movable point source (sect.4c) is also schematically drawn: the source, moving inside a steel tube, traverses all the towers at a fixed longitudinal depth along scintillator centerlines.

3: Quadrant of the calorimeter where A,B,C show Central, Endwall and Plug respectivly. Towers are numbered from 0 (at $90^{\circ}$ in polar direction) to 11 (last tower of Endwall modules). Hadronic towers 6,7 and 8 are shared between Central and Endwall calorimeter.

4: Two WLS strips collect light from each scintillator layer. In the air gap between WLS and light guide, filters are inserted to insure the light output equalization for different layers of the same tower.

5: Gain variation of a 9954 p.m., as the anode current $I_{a}$ is changed at $t=t_{0}$ from 0 to $\sim 750 \mathrm{nA}$.

6: Response of a scintillator, when irradiated by a $\mathrm{Sr}^{90} \beta$ source. It was read out by a single wave-length shifter along a short side corresponding to a fixed azimutal angle (constant $y$ ). The scintillator had tapered edges in (a) and untapered in (b).

7: Response map of the same scintillator as in Fig. 6a, but read by WLS positioned at $\theta=$ constant as shown in fig 4 . The displayed values are the sum of pm1 and pm2 .

8: Response of an Endwall tower, when irradiated by the "skin source".Peaks correspond to the passage of the source in front of one layer of the tower. Coupling scintillators to light guides, selected according to their attenuation length, is not sufficient to get uniformities of better than $15 \%$ (Fig. $8 a$ ). The result in Fig. $8 b$ is achieved by inserting filters in the air gap between WLS and light guides. 
9: Layout of the laser system.

10: Laser light distribution disc.

11: Muon spectrum for an Endwall tower. The peak corresponds to about 2 $\mathrm{GeV}$ energy deposition for a hadronic cascade.

12: Linearity plot of tower 5 for pion energies ranging from 10 to 150 GeV.

13: Pulse height distribution for $50 \mathrm{GeV}$ pions in Central tower 1. Only pions which are m.i.p. in the e.m. compartment are plotted.

14: Pulse height distribution for $50 \mathrm{GeV}$ pions in Endwall tower 10. Pions have been selected as in Fig. 13.

15: Pulse height distribution for Central tower 1. Pions interacting in the e.m. compartment are included and pulse height is the sum of hadron and electromagnetic calorimeter signals.

16: Energy resolution as function of pion energy for towers 1,5 and 10.

17: $\theta(\eta)$ response of the calorimeter. The ratio between the measured and the incident energies is shown for different beam positions expressed as function of $\eta=-\ln (\tan \theta / 2)$. The beam was centered in $\phi$.

18: Fraction of the total energy deposited in neighbouring towers as function of the tower number. Beam was hitting the tower centers.

$19: \phi$ response to a $50 \mathrm{GeV}$ pion beam sweeping the facing towers of two stacked modules. The beam was aimed at the tower center in $\theta$ and had a radius $\sim 2 \mathrm{~cm}$.

20: The logarithm of the ratio of the left (L) and right (R) p.m. pulse heights in a tower, as function of the distance of the particle from the tower center. The dimensions refer to a scintillator located at a longitudinal depth close to the shower maximum.

21: Position resolution as function of the distance from tower center for different pion energies.

22: Fine $\phi$ scan of the interface region between two modules. Each point is the average of the energy in a $1 \mathrm{~mm}$ bin.

23: Observed "hot spot" pulse height as function of the $e^{*}$ energy and for different thickness of the crack radiator. Response is linear as function of energy and is reduced by a factor $\sim 10$ by 10 radiation lengths of absorbing material.

24: The $\sigma$ of the ratio of $L$ and $R$ p.m.'s in a tower as function of the incoming pion energy, for particles hitting the tower away from the crack region.

25: 2-dimensional plot of the response of modules 1 and 2 to a $50 \mathrm{GeV} \mathrm{e}$ 
beam.

26: The same data as in Fig. 25 after a $3 \sigma$ cut on the L/R ratio.

27: Percentage difference between corresponding pion peaks of 7 recalibrated modules. The peaks values are normalized to the reference $\mathrm{I}_{\mathrm{Cs} 137}$ currents.

28: Percentage difference between the $l_{c}$ currents of each p.m. and the mean value of the $I_{c}$ 's corresponding to towers with the same number. On each p.m. the $I_{c}$ 's were scaled, via $I_{C_{s 137}}$, to give a pion calibration of $2 \mathrm{pC} / \mathrm{GeV}$.

29: Distribution of percentage differences of $I_{\mathrm{Cs} 137}$ currents in Endwall tubes, when measured in the presence of no magnetic field and with the superconducting solenoid at $15 \mathrm{kG}$, representing combined scintillator, WLS and p.m. response changes.

30: Percentage differences of the charges produced by Endwall p.m.'s exposed to light from the laser system, when measured with the magnetic field "on" (15 kG) and "off".

31: Difference, tube by tube, of the quantities plotted in figures 29 and 30 , representing response change of scintillator plus WLS only.

32: Change of the attenuation length $\lambda$ of a scintillator exposed to increasing radiation doses. 


\section{Tables}

1: Various parameters of central hadron calorimeter.

2: Various parameters of endwall hadron calorimeter.

3: Detected energy versus tower number for traversing muons. The energy is normalized to the detected energy of $50 \mathrm{GeV}$ pions. 


\section{References}

[1]: Manufactured by Polivar Spa, Pomezia (Italy).

[2]: Manufactured by Exciton Inc., (U.S.A.)

[3]: Manufactured by Roehm Gmbh, Darmstadt (BRD)

[4]: Manufactured by Thorn EMI Ltd. ,Middlesex (U.K.).

[5]: "The Rabbit system: low cost, high reliability, front end electronics featuring 16 bit dynamic range", NIM, this volume.

[6]: CDF Design Report, August 1981,unpublished.

[7]: H. Jensen et al., CDF Note \# 185, unpublished

[8]: Manufactured by Lambda Physik, Goettingen (BRD).

[9]: Manufactured by Harshaw, Solon (Ohio, USA).

[10]: "Cosmic ray test of the CDF Central calorimeters", NIM, this volume.

[11]: M.J. Corden et al., NIM , A238(1985)273; Physica Scripta 25(1982)5; Physica Scripta 25(1982)11.

[12]: S. Kuhlmann et al., CDF Note \#413, unpublished

[13]: S. Bertolucci et al., NIM 25A(1987),561

[14]: The NW Wedge Group consists of:

J. Cooper,A. Hahn, S. Hahn, M. Miller, H. H. Williams, R. Diebold, L. Nodulman, J. Proudfoot, P. Schoessow,D. Underwood, R. Wagner, A. Wicklund, T. Kamon, A. Yamashita, S. Mikamo, K. Chadwick, J. Simmons, S. Cihangir,D. Smith, T. Westhusing, $H$. Jensen and K. Yasuoka 


\section{TABLE 1}

\section{Parameters of the Central hadron Calorimeter}

Modules

Number of modules

48

Length

Width (in $\phi$ direction)

$2.5 \mathrm{~m}$

Weight per module

$1.33 \mathrm{~m}$.

$12,000 \mathrm{~kg}$.

Towers

Total number ( $8 /$ module)

384

Length $\left(\Delta \phi=15^{\circ}\right)$

0.56 to $0.91 \mathrm{~m}$.

Width $(\Delta \eta=0.11)$

Total depth (hadron calorimeter alone) 0.28 to $0.45 \mathrm{~m}$

$4.7 \wedge_{\text {abs }}$

\section{Layers}

Number

Steel thickness

Scintillator thickness

Scintillator type

Wave shifters

Number of phototubes
32

$2.5 \mathrm{~cm}$

$1.0 \mathrm{~cm}$

PMMA doped with $8 \%$ Naphtalene

$1 \%$ Butyl-PBD and $.01 \%$ POPOP UVA PMMA doped with $30 \mathrm{mg} / \mathrm{l}$ Laser dye \#481. 


\section{TABLE 2}

\section{Parameters of the End Wall hadron Calorimeter}

Modules

Number of modules

48

Approximate dimensions

$0.8 \times 1.0 \times 1.1 \mathrm{~m}^{3}$

Weight per module

$7,000 \mathrm{~kg}$.

\section{Towers}

Total number ( $6 /$ module)

288

Length $\left(\Delta \phi=15^{\circ}\right)$

0.35 to $0.78 \mathrm{~m}$.

Width $(\Delta \eta=0.11)$

0.25 to $0.40 \mathrm{~m}$

Total depth (hadron calorimeter alone)

$$
4.5 \wedge_{\text {abs }}
$$

\section{Lavers}

Number

Steel thickness

15

Scintillator thickness

Scintillator type

$5 \mathrm{~cm}$

$1.0 \mathrm{~cm}$

PMMA doped with $8 \%$ Naphtalene

Wave shifters $1 \%$ Butyl-PBD and .01\% POPOP UVA PMMA doped with $30 \mathrm{mg} / \mathrm{l}$ Laser dye \#481

Number of phototubes 


\section{TABLE 3}

TOWER \#

0

1

2

3

4

5

6

7

8

9

10

11
MUON/PION *50 (GeV)

1.85

1.85

1.92

2.02

2.15

2.41

1.90

2.18

2.06

2.34

2.13

2.14 


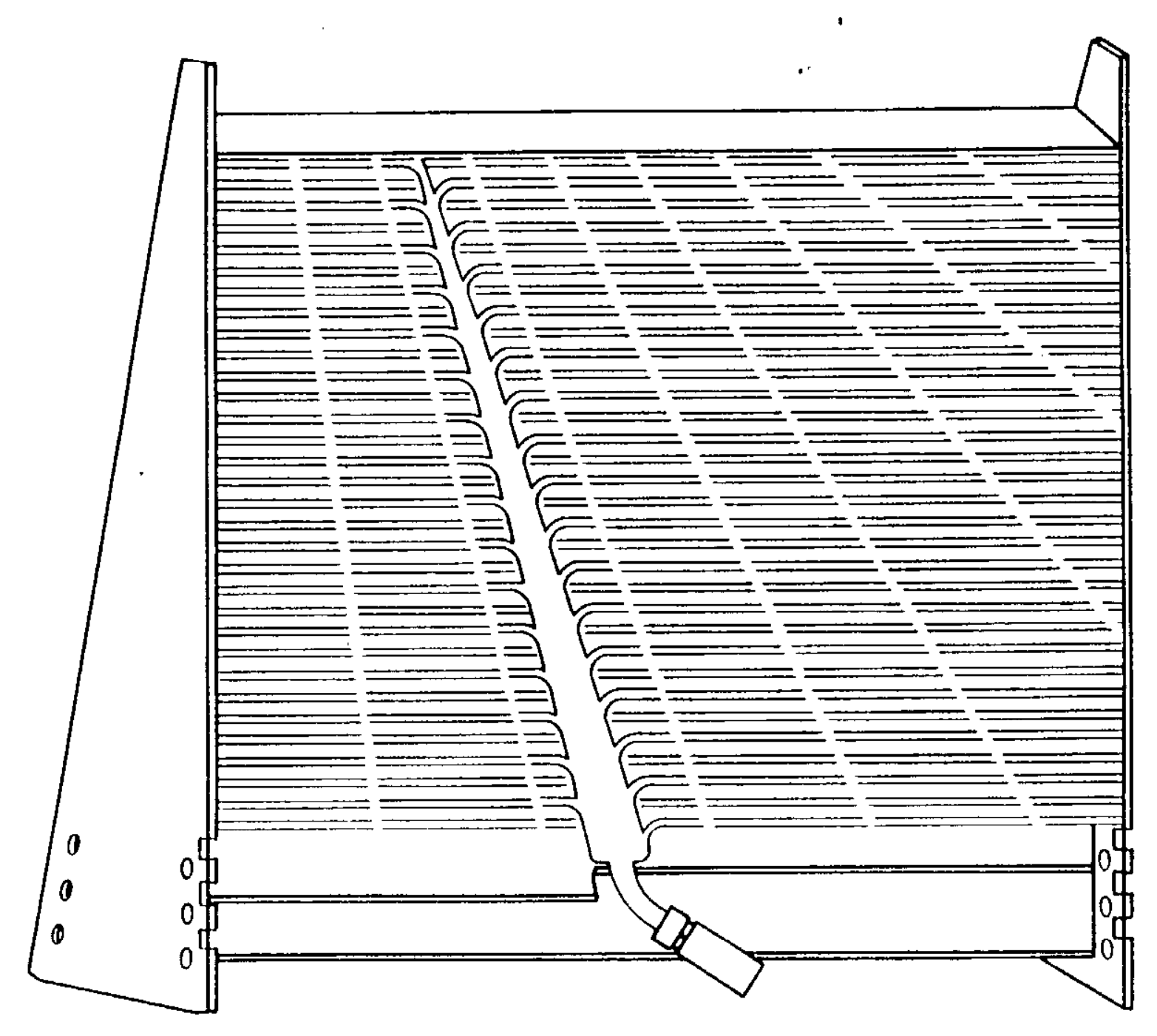




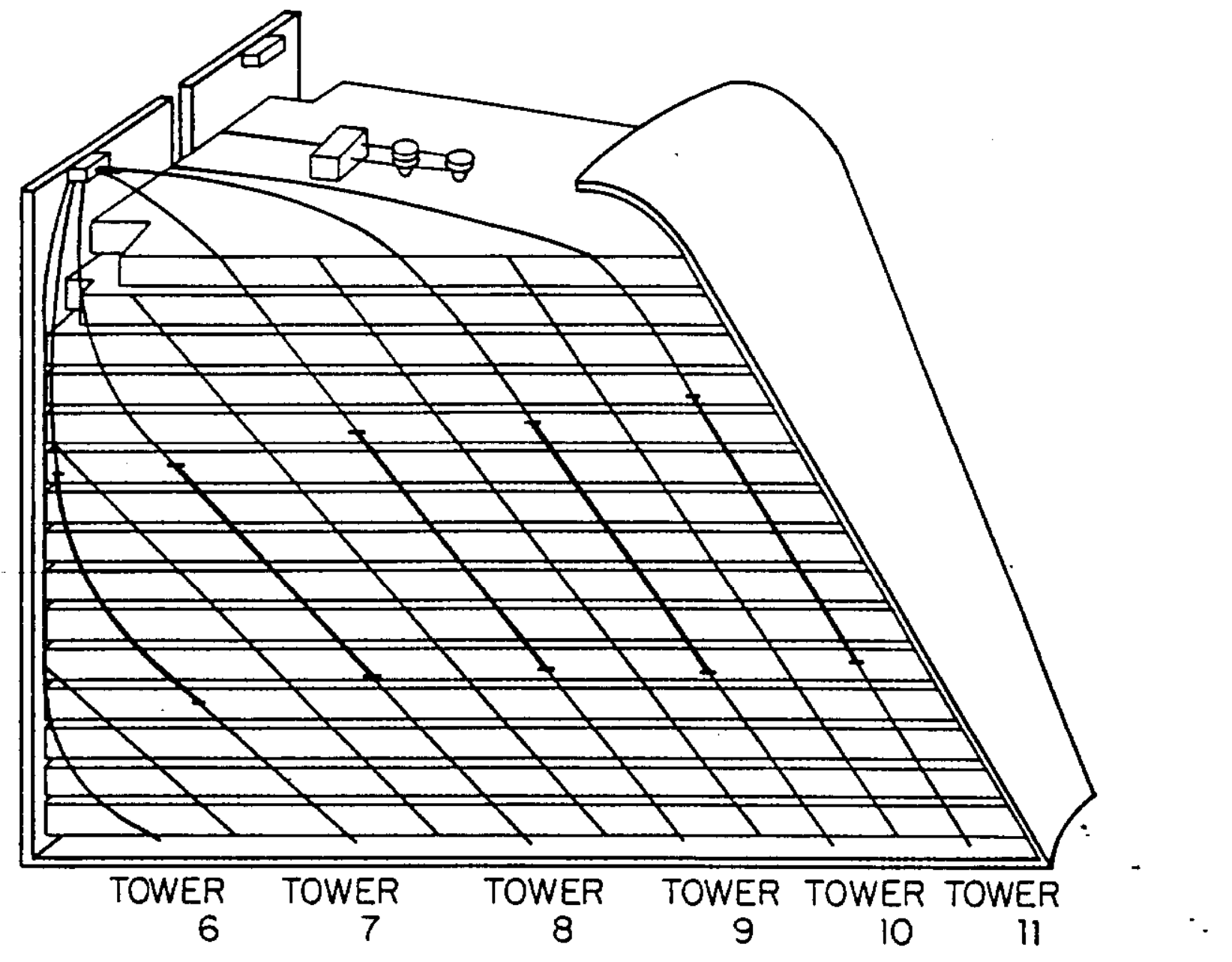

Fig 2 


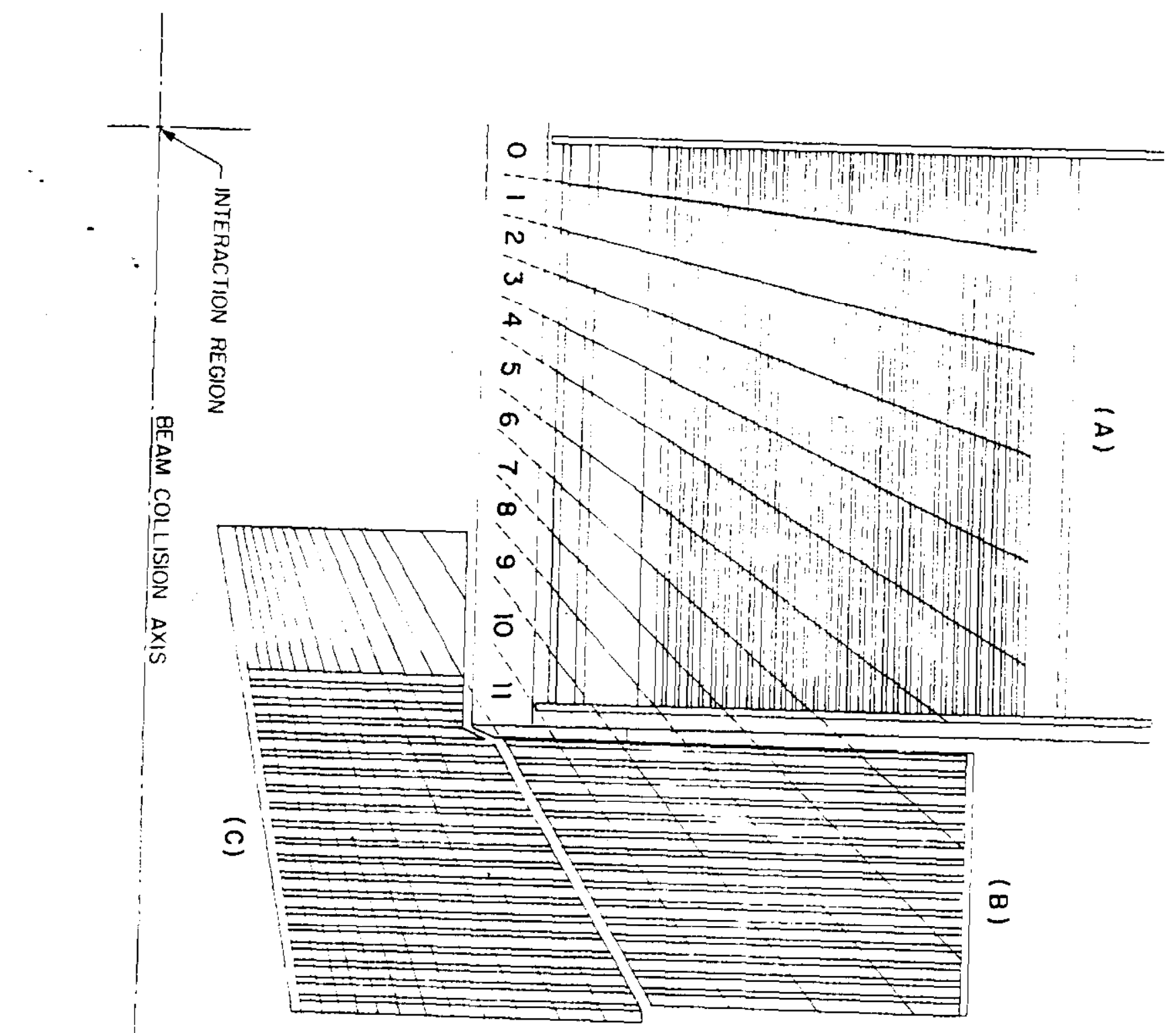




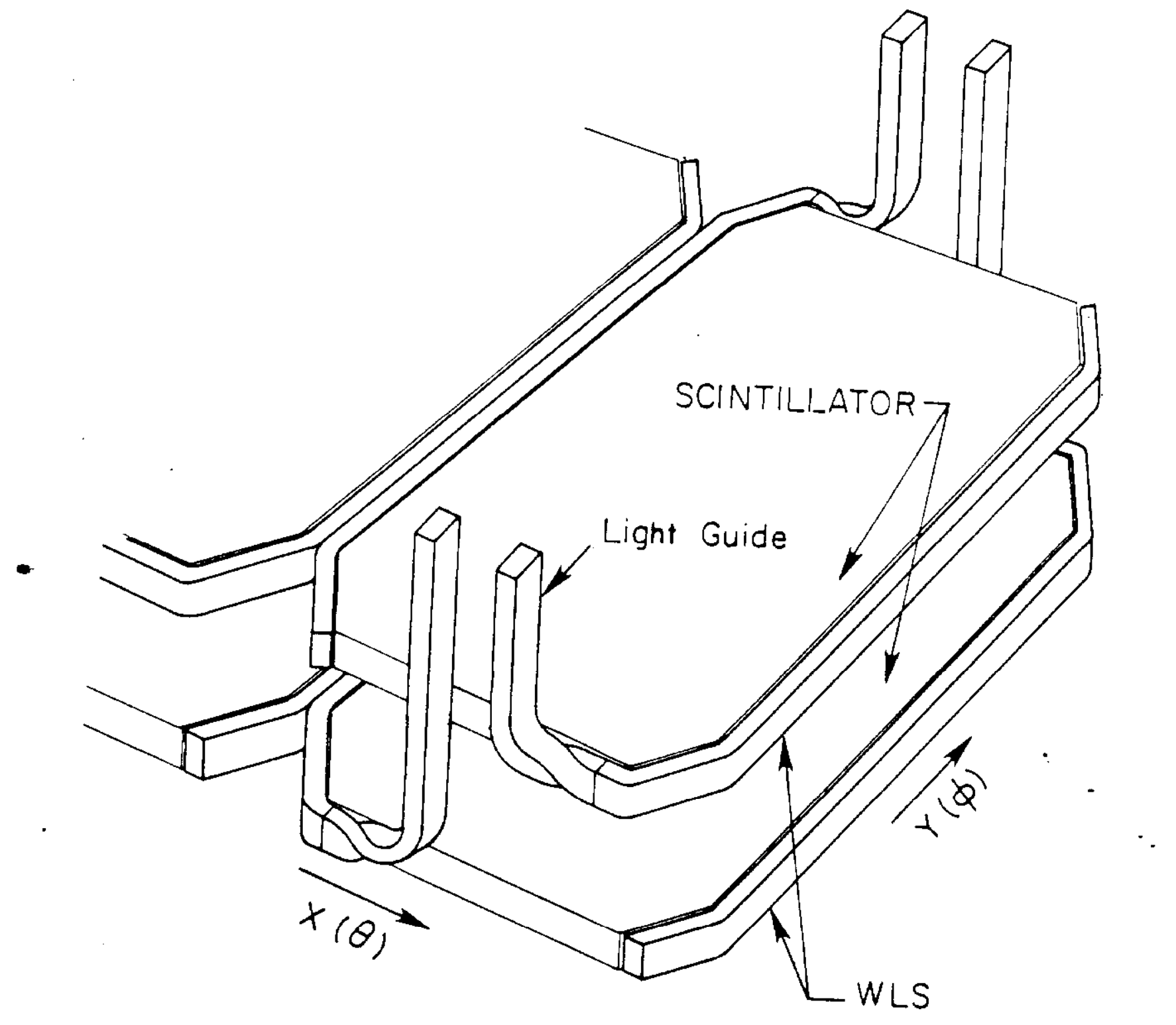

$$
F . S \quad
$$




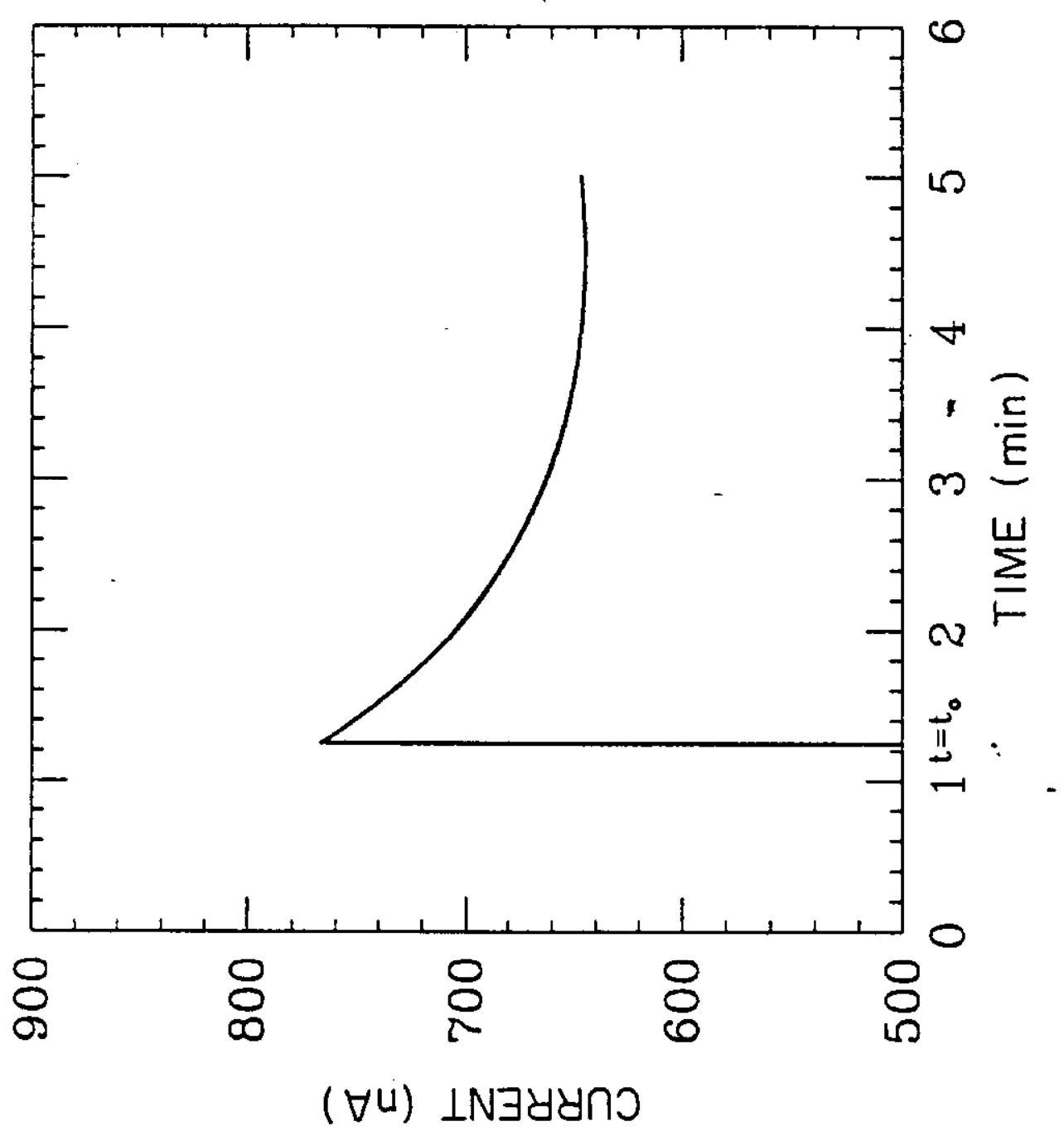


$F_{\text {Ig }} 6 a$

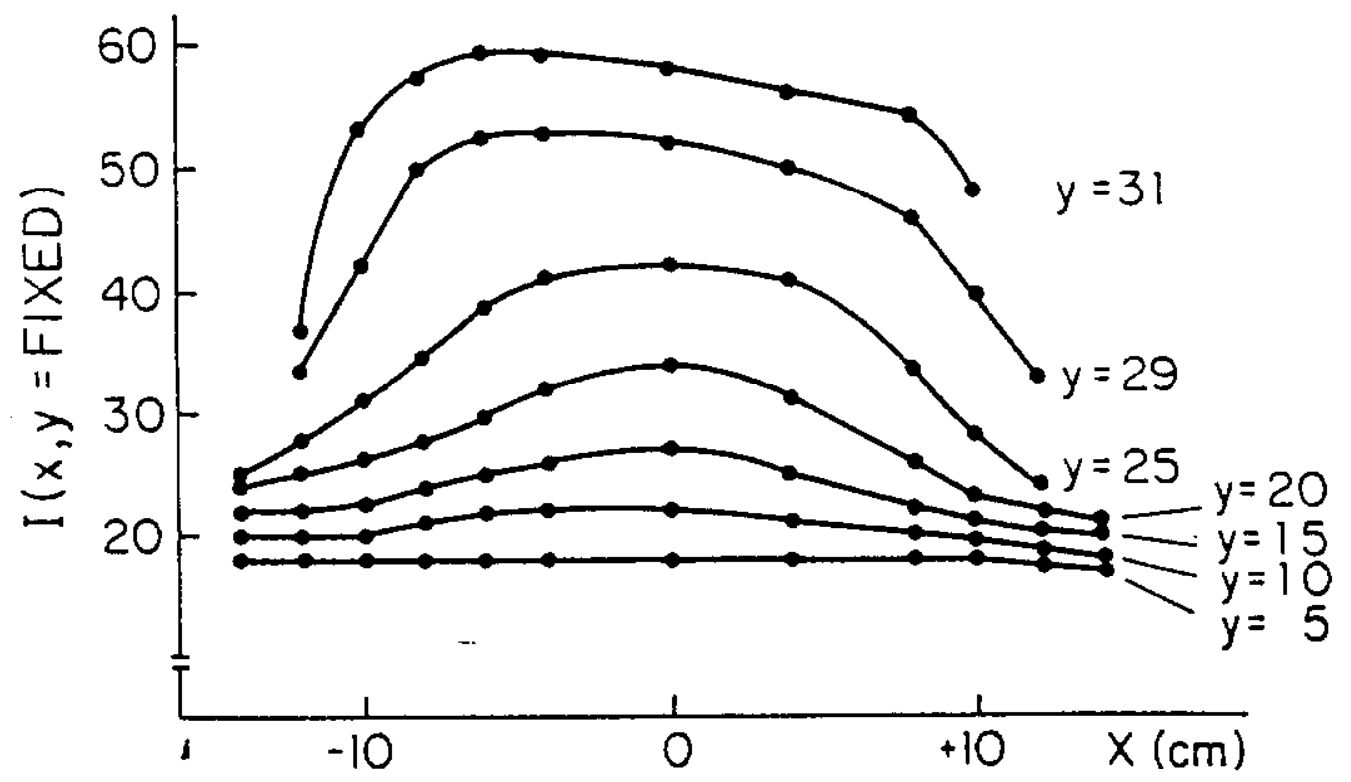

Fig $6 b$

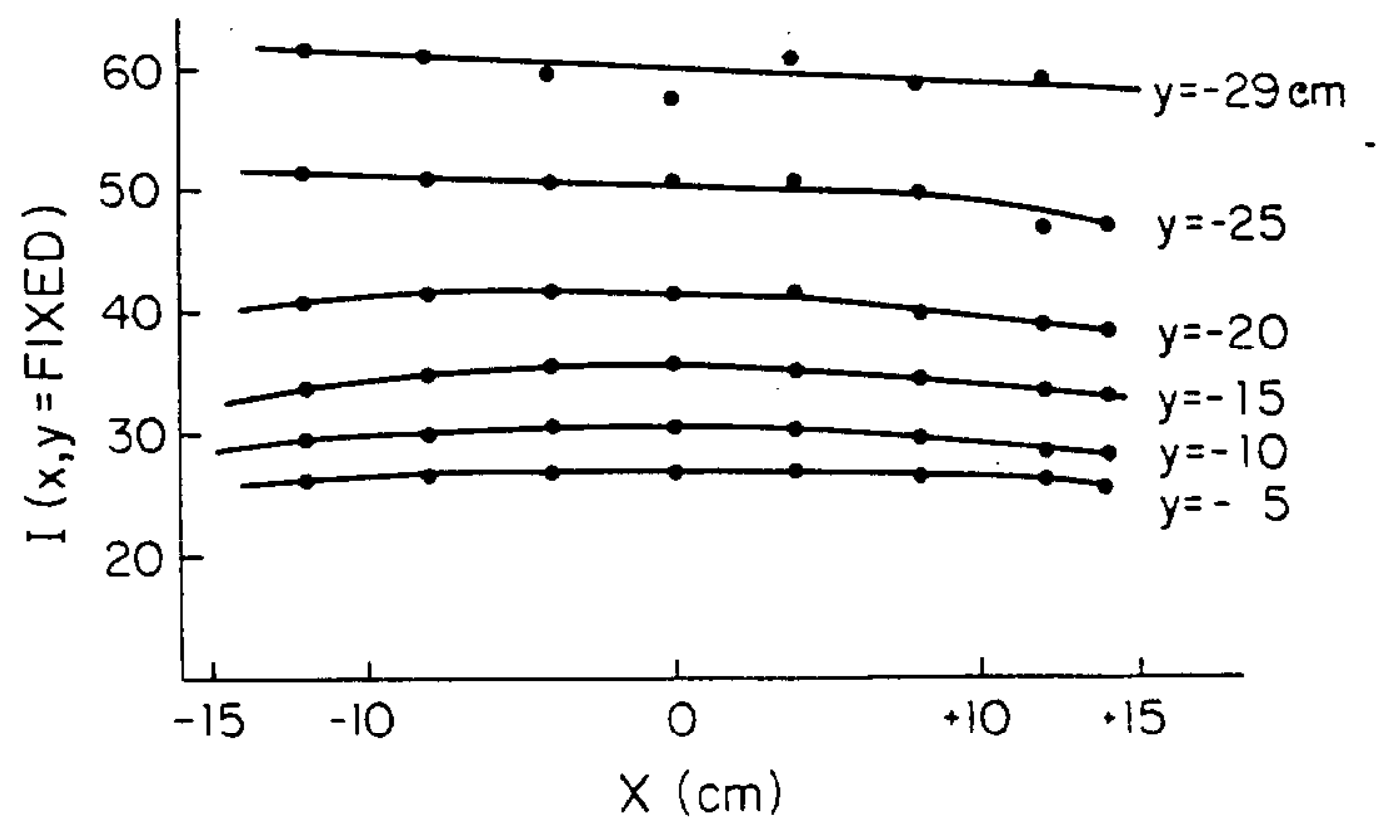




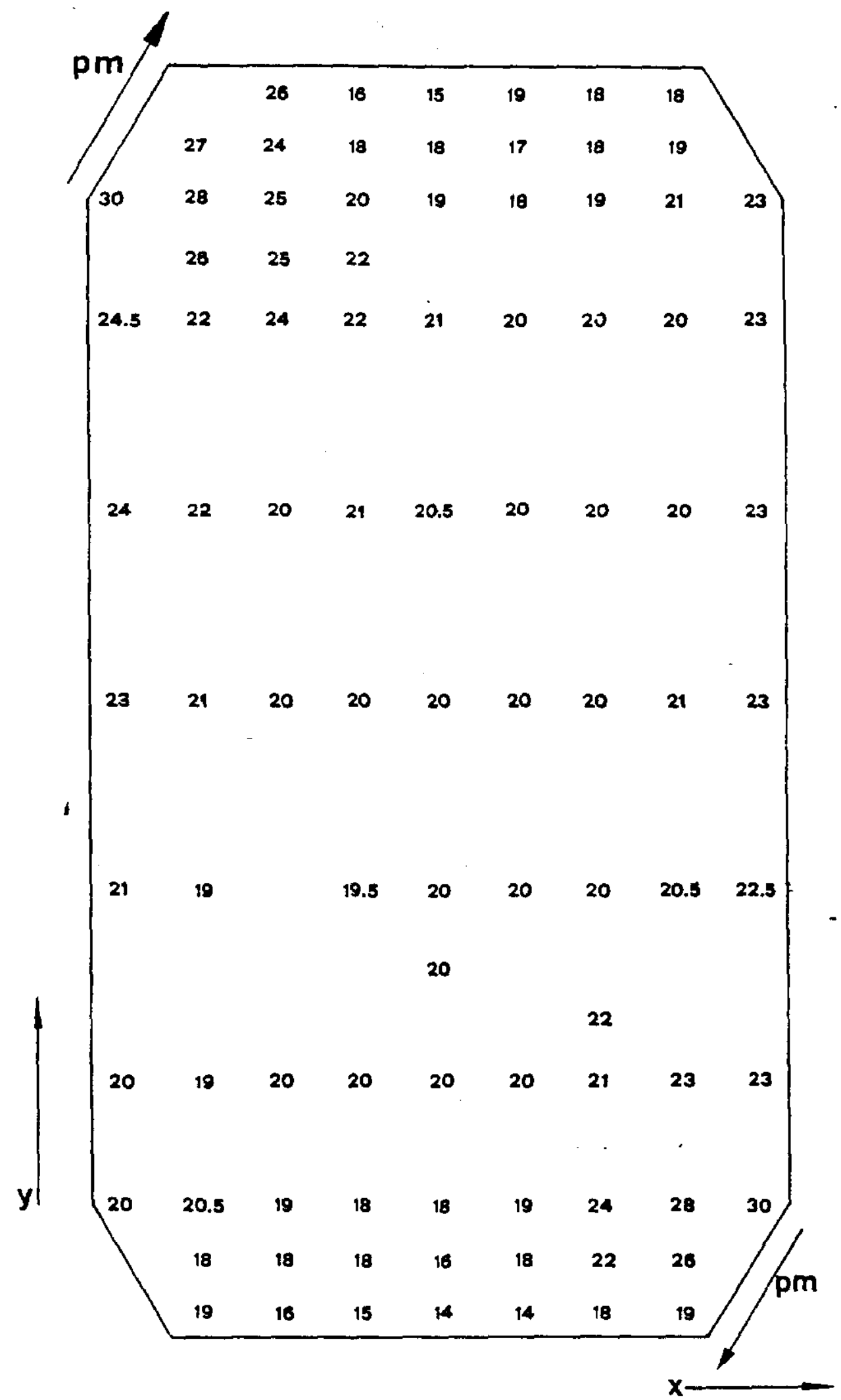




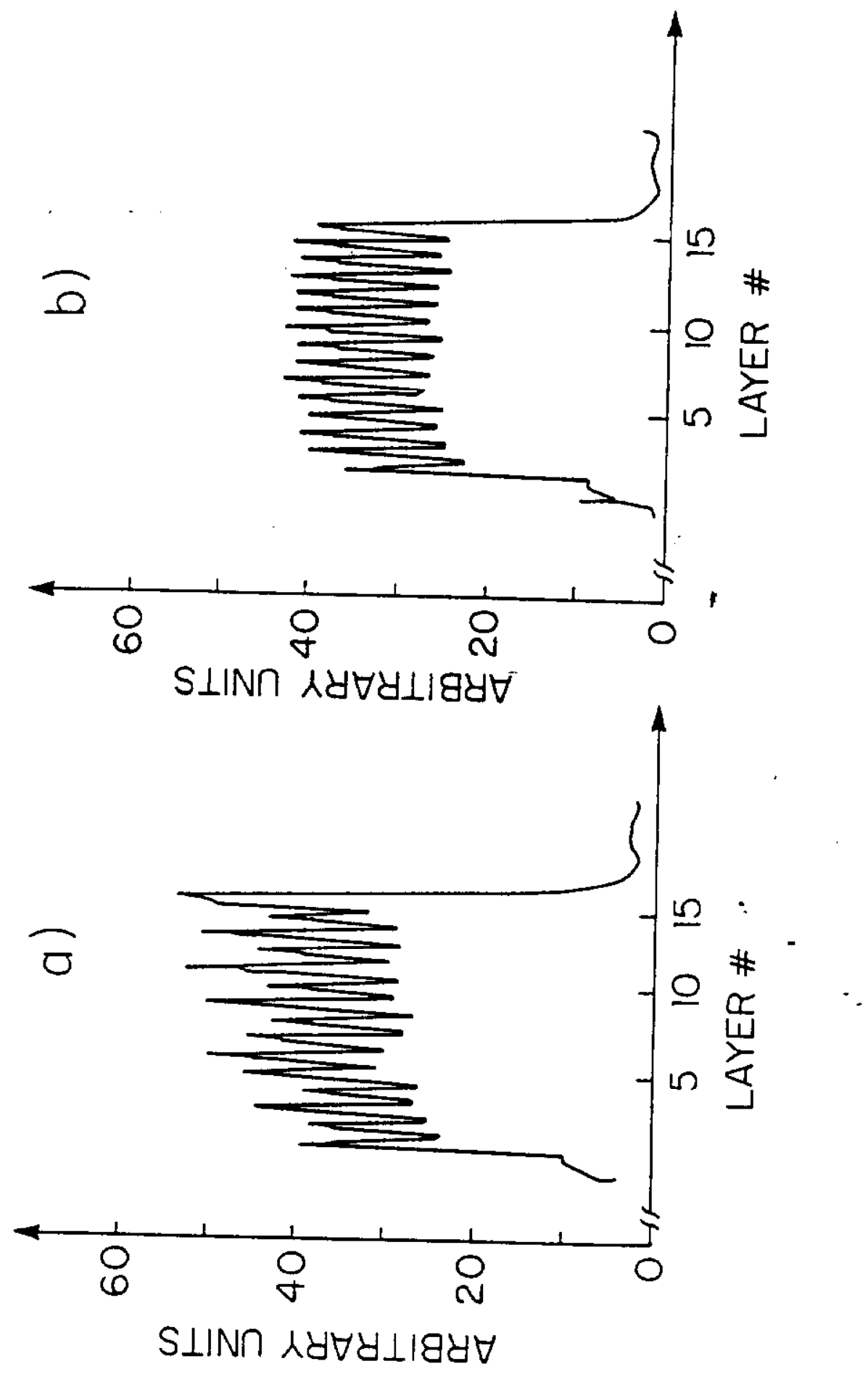




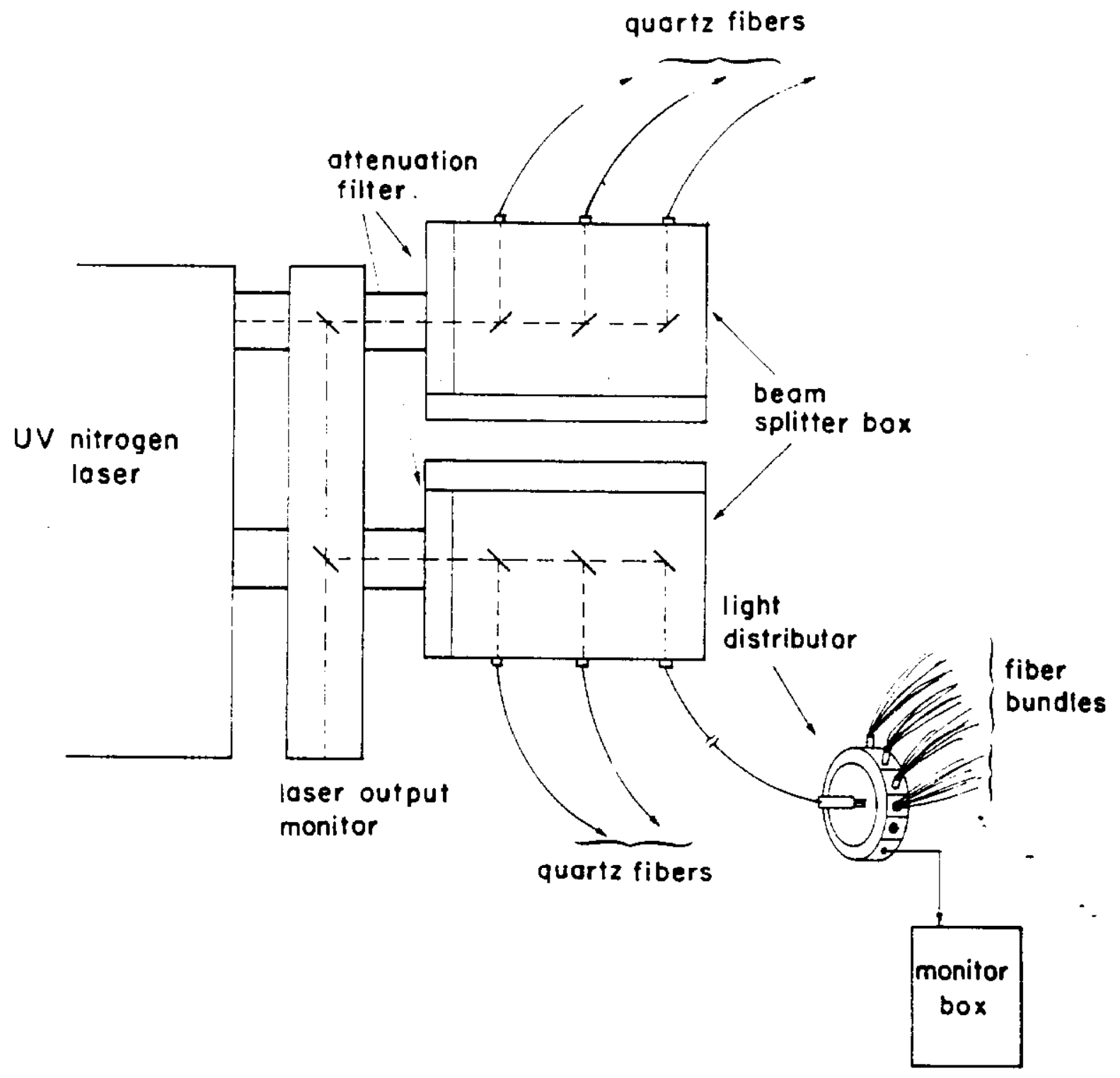

$F \cdot g$ 


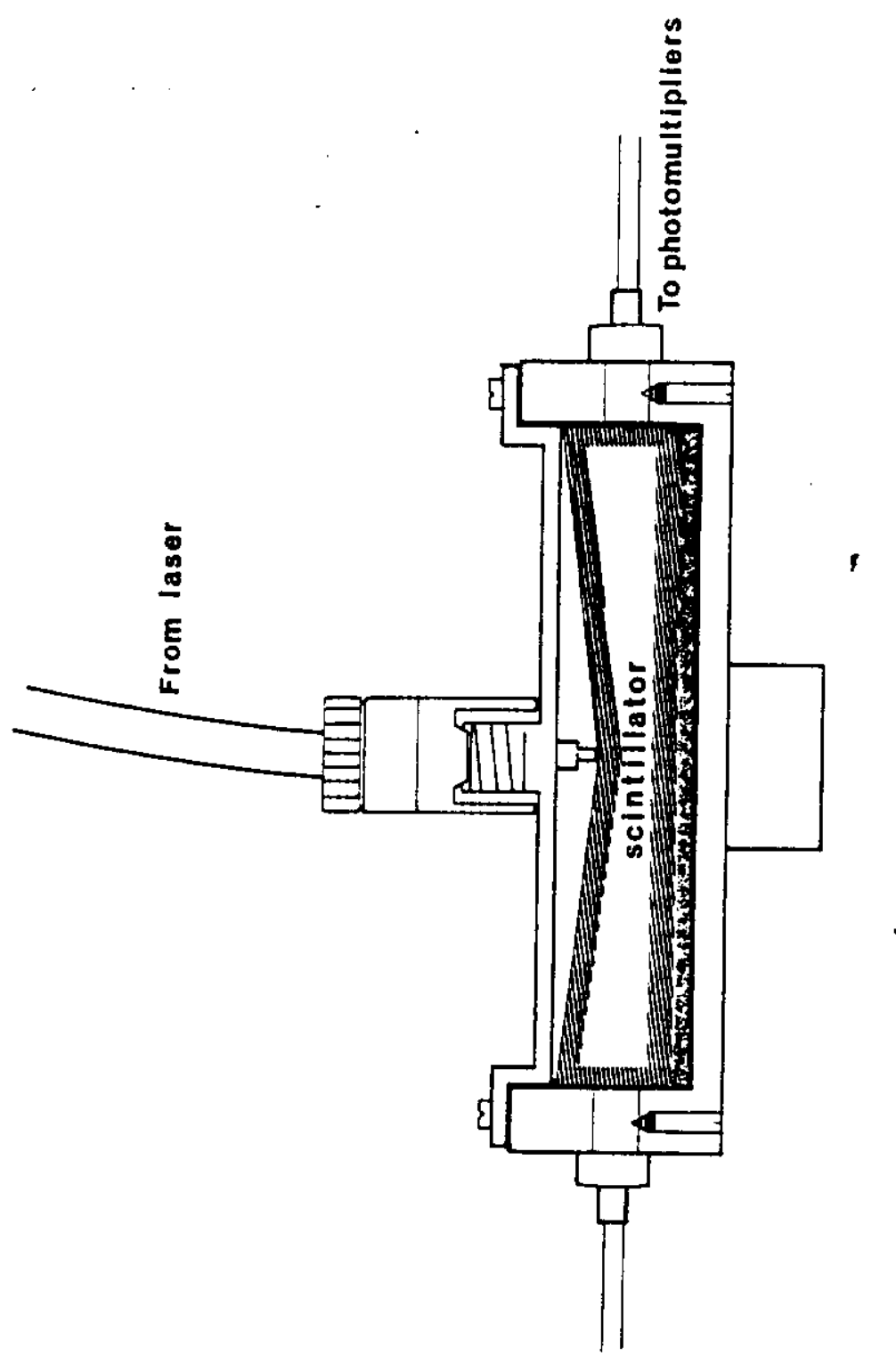



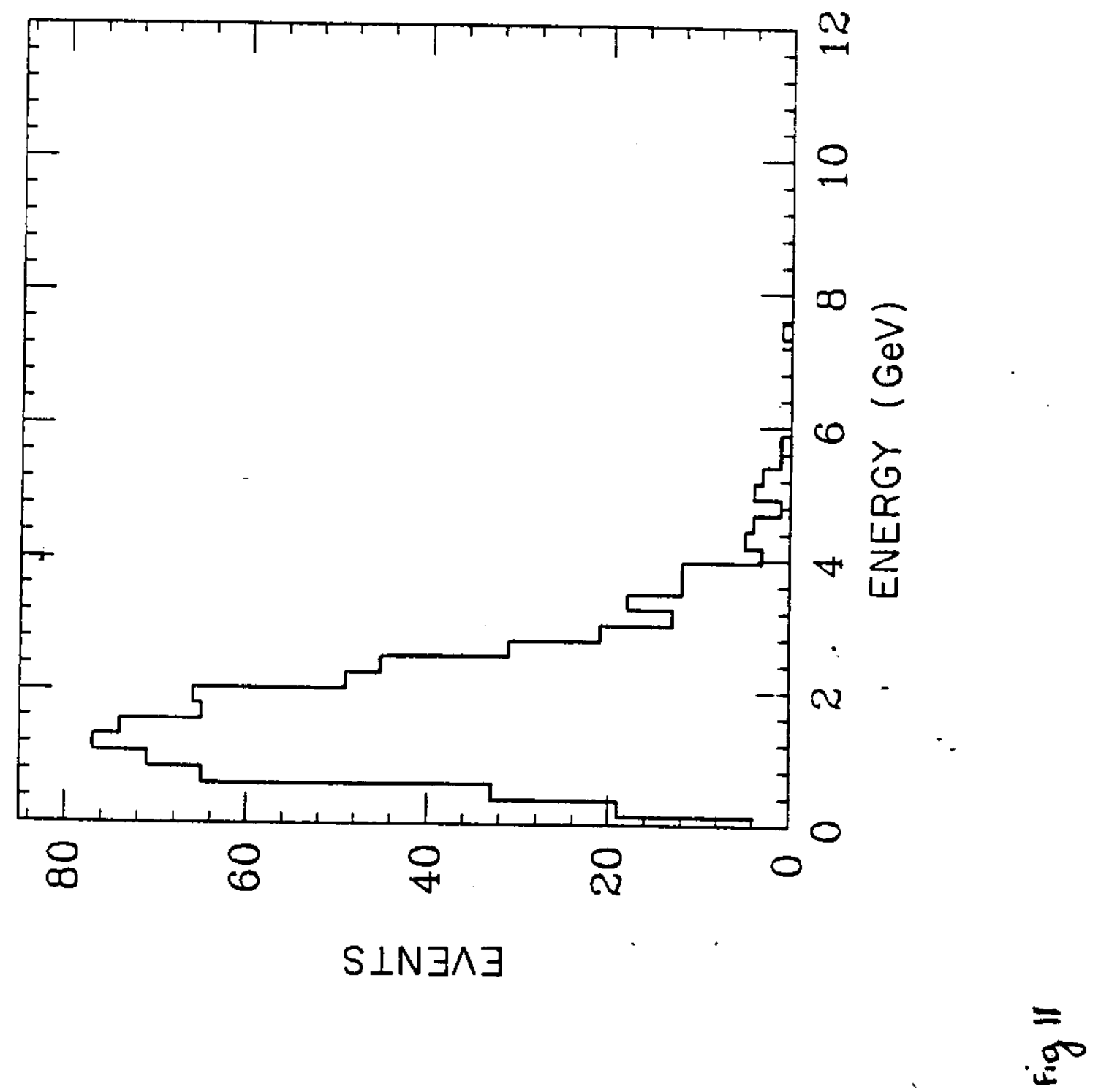
$\frac{\pi}{5}$

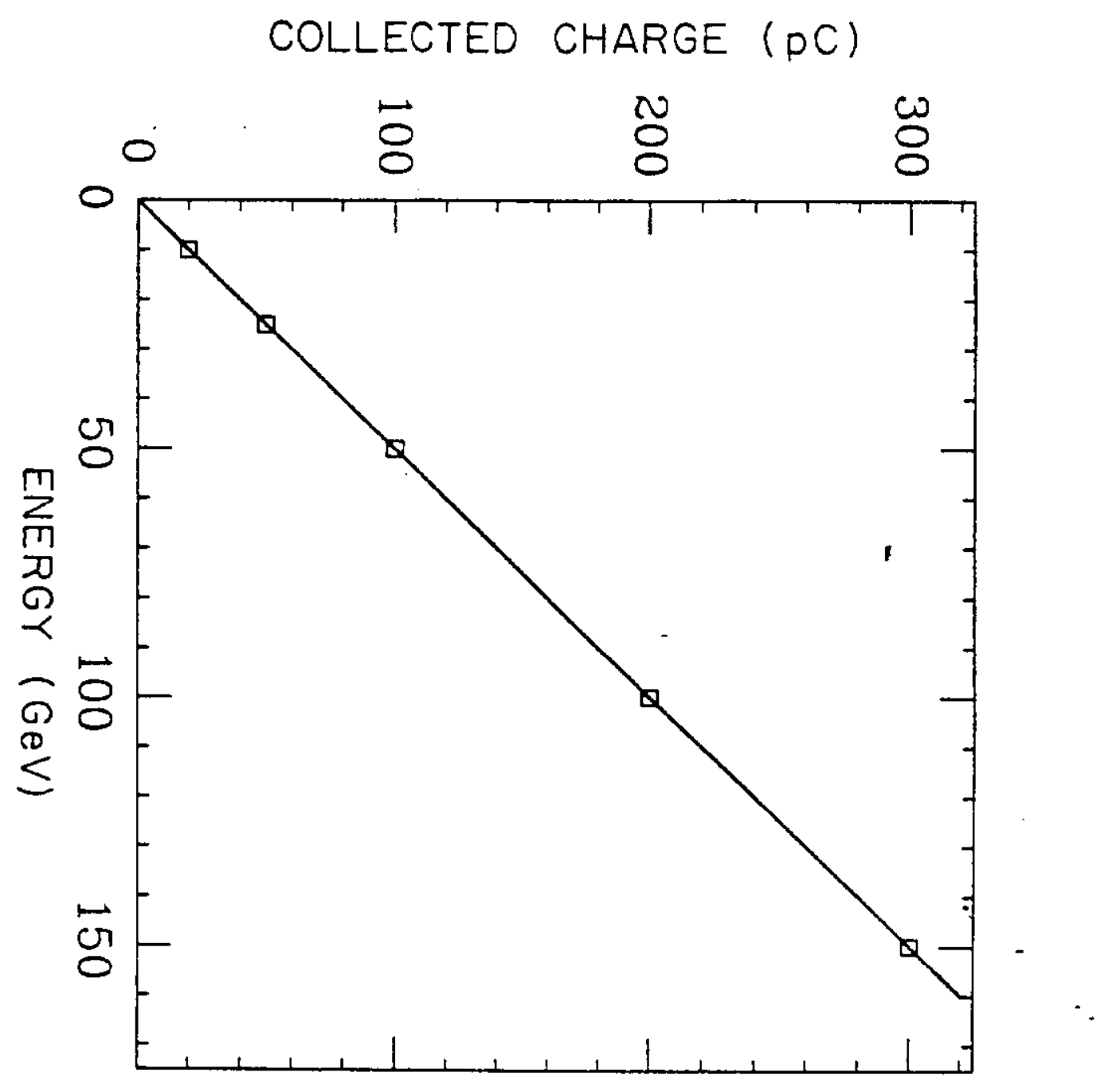


$\stackrel{20}{\omega}$

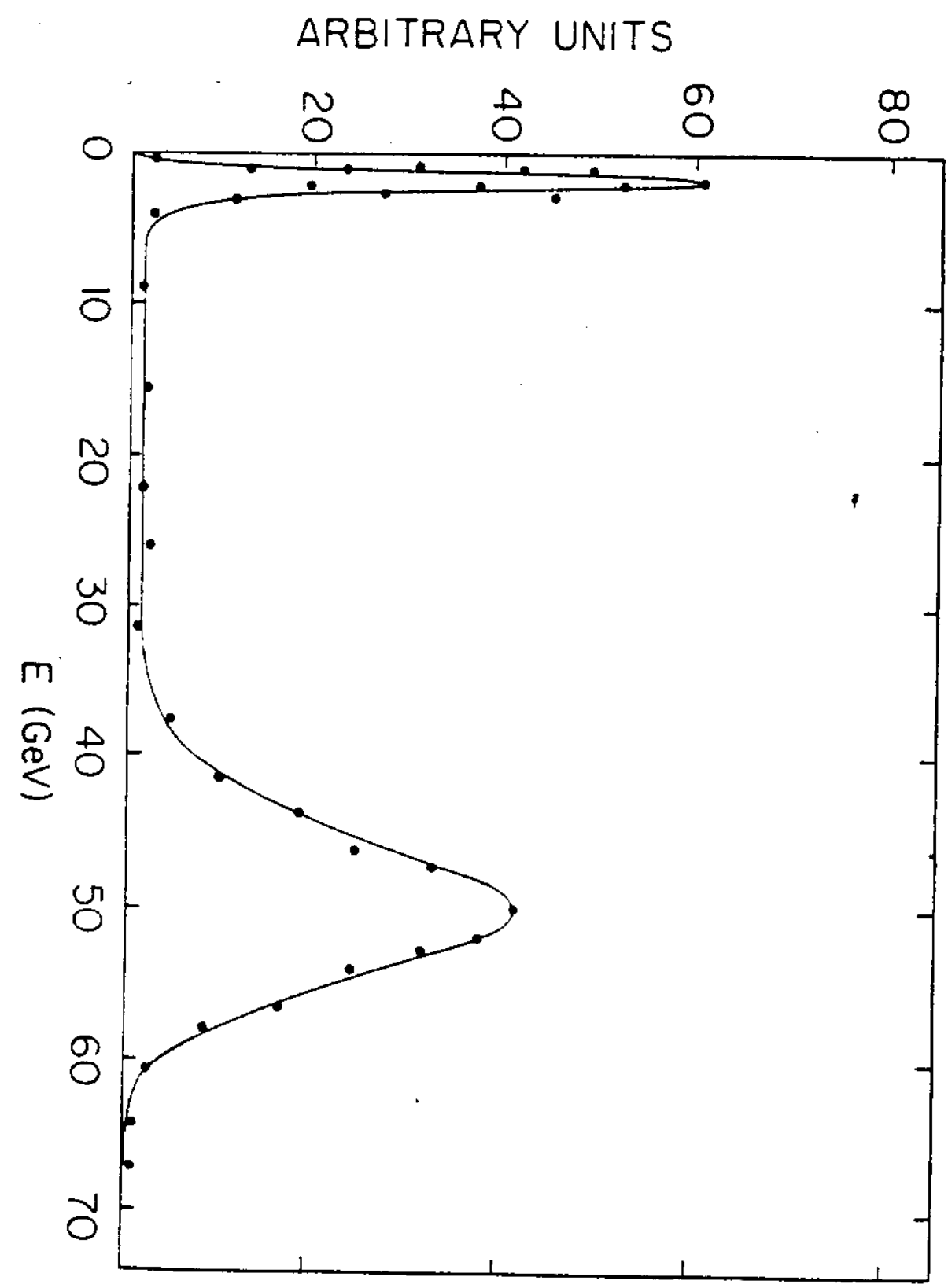




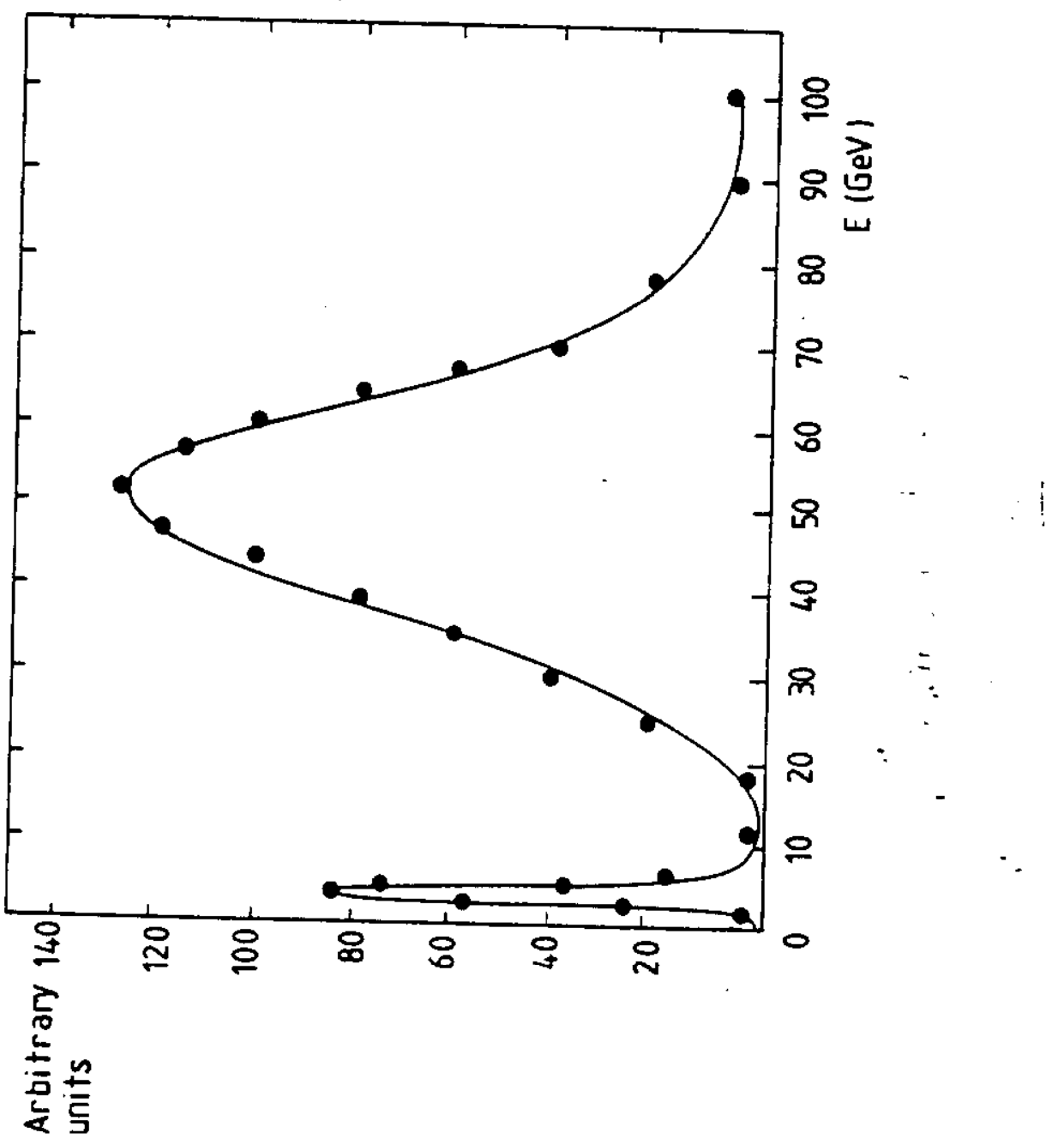




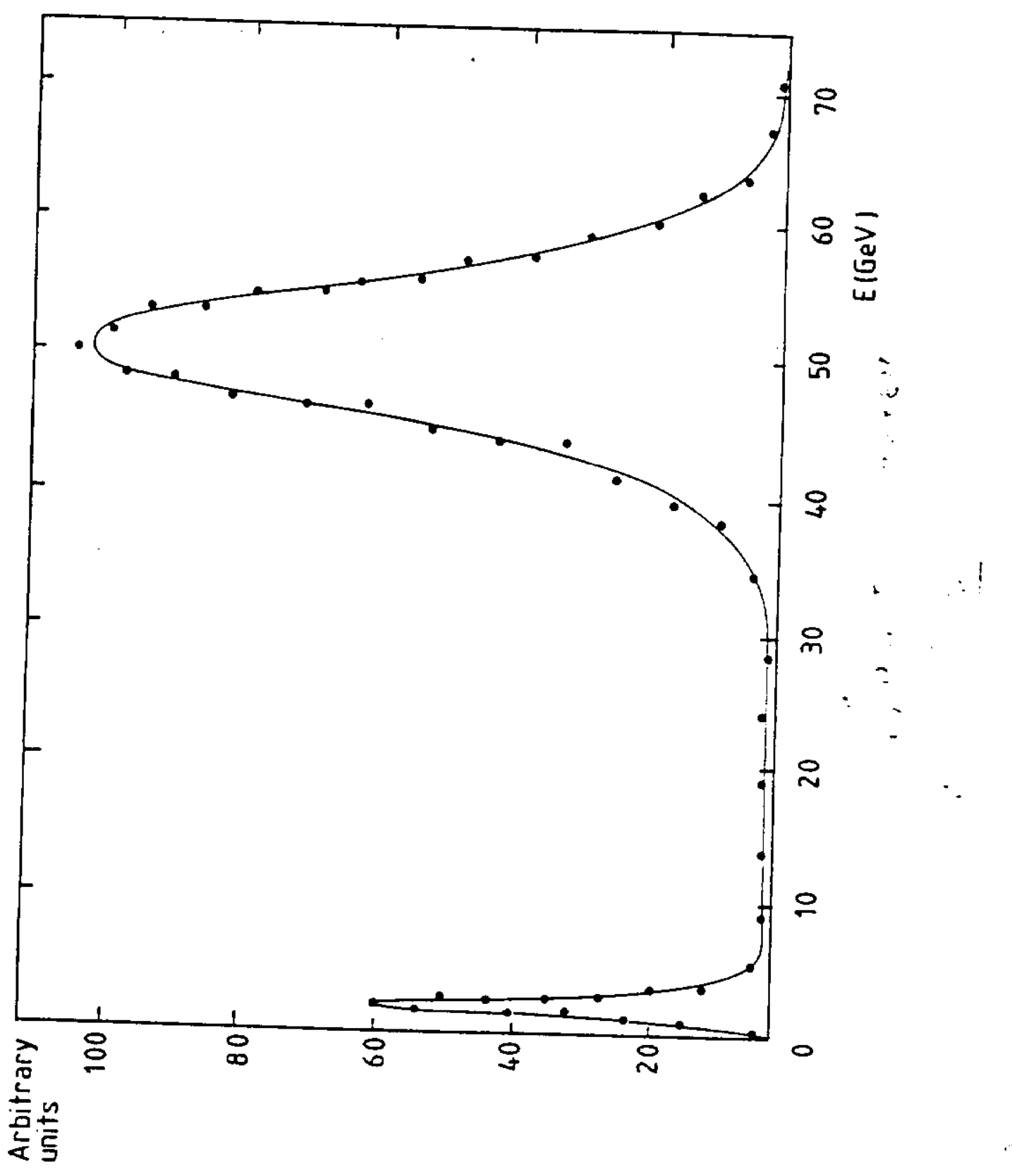

$\frac{n}{\pi}$ 
Resolution (\%)

i!
un

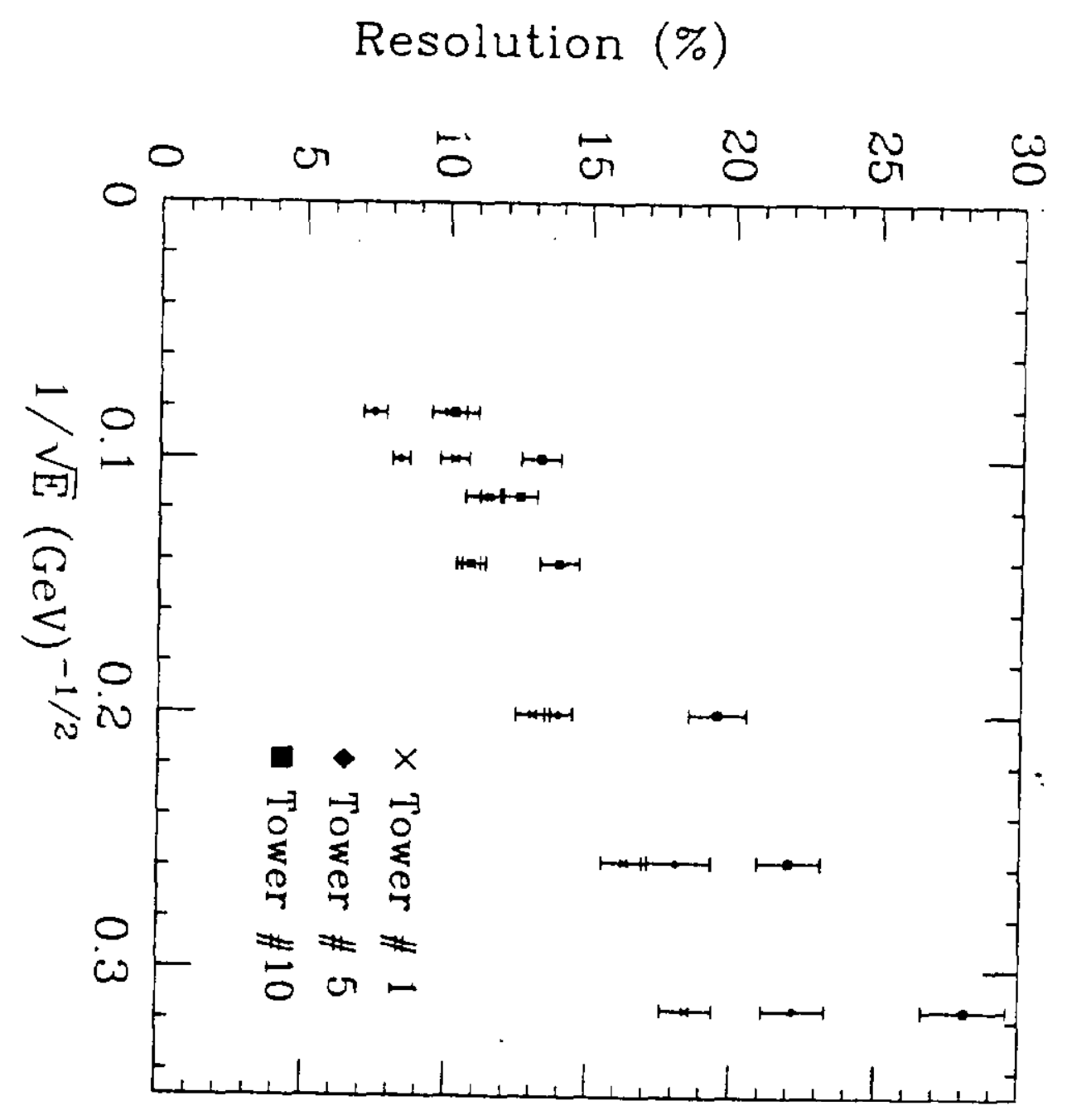




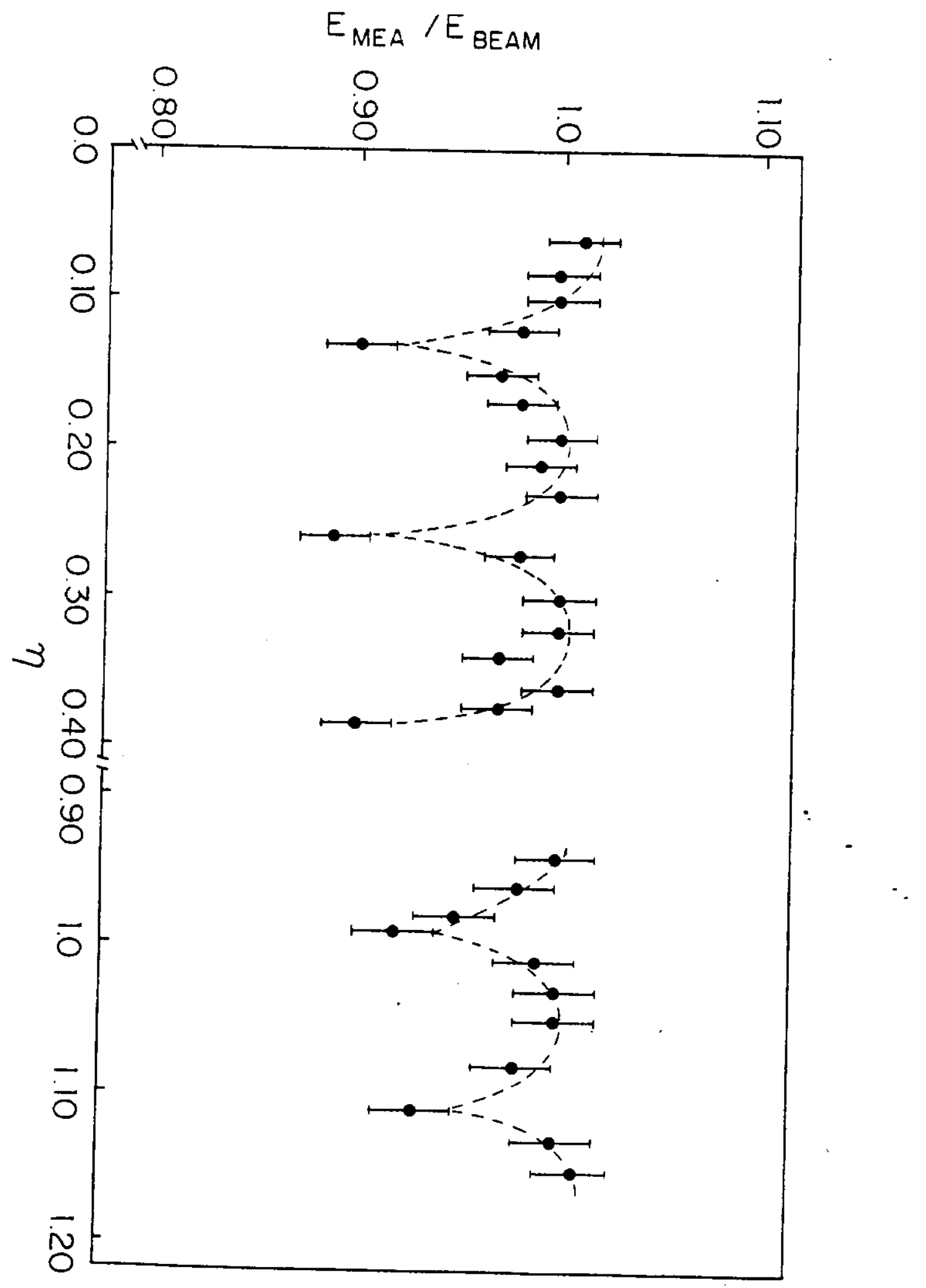




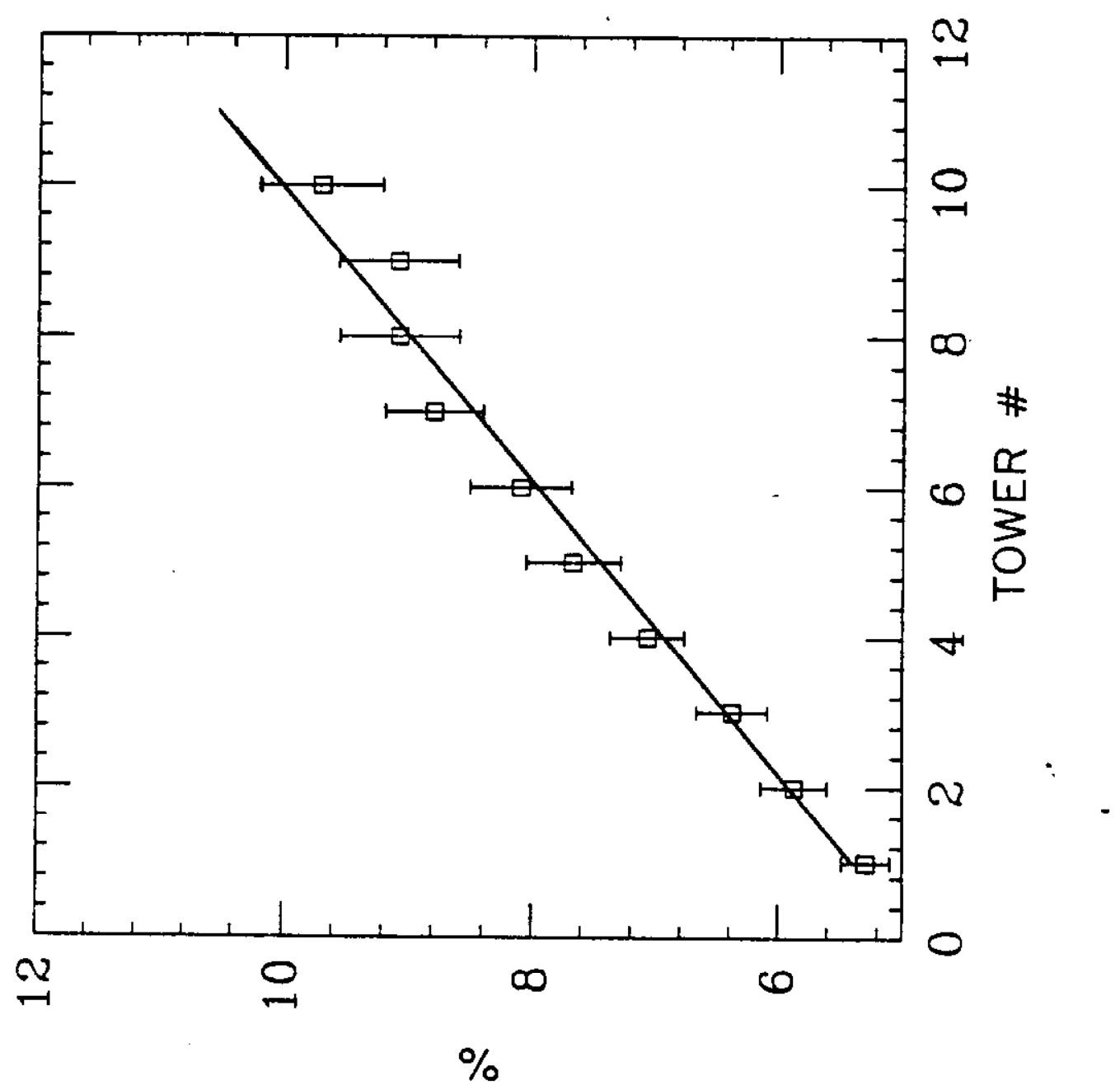

$\underline{\infty}$
$\stackrel{\sigma}{L}$ 


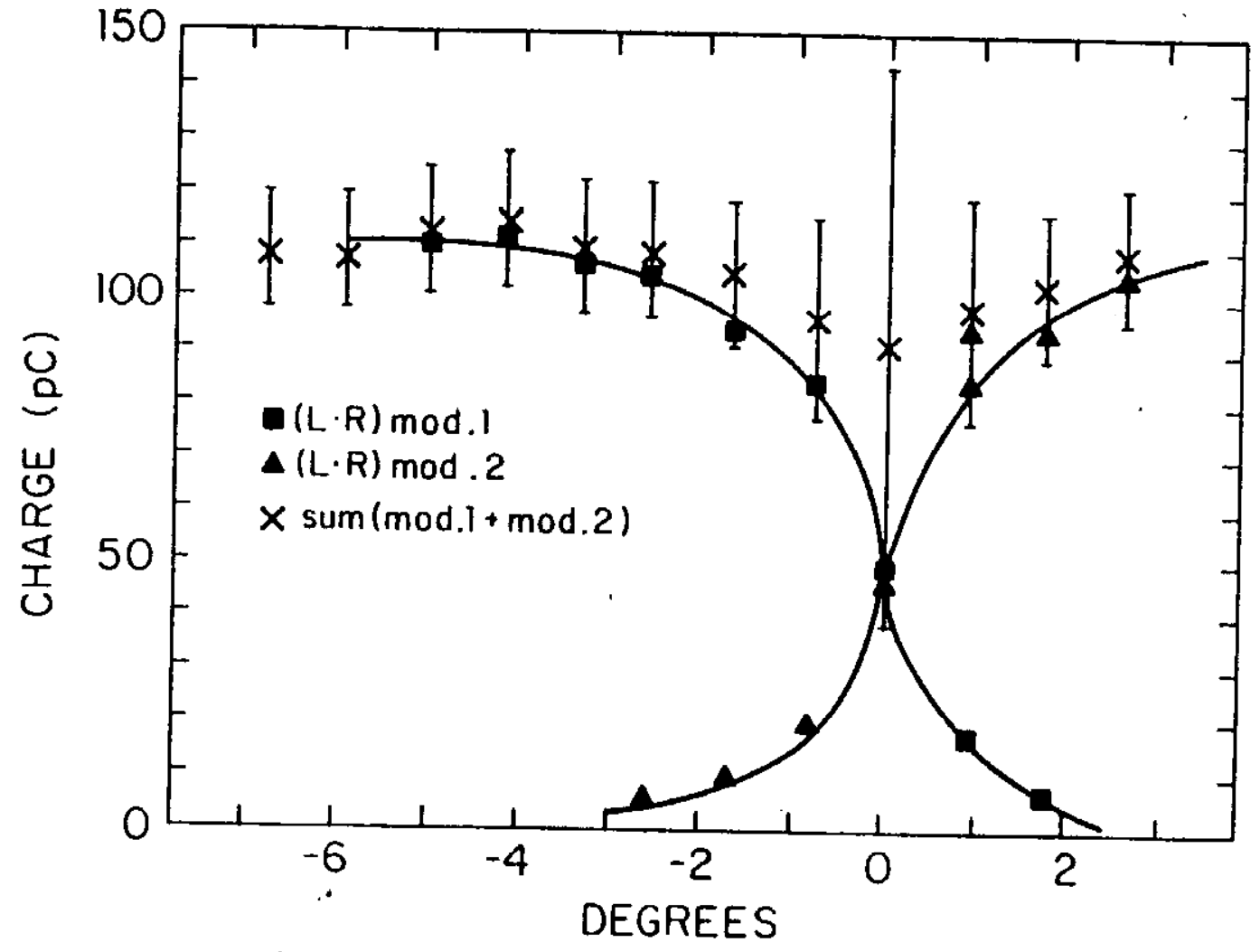

$F_{19} 19$ 


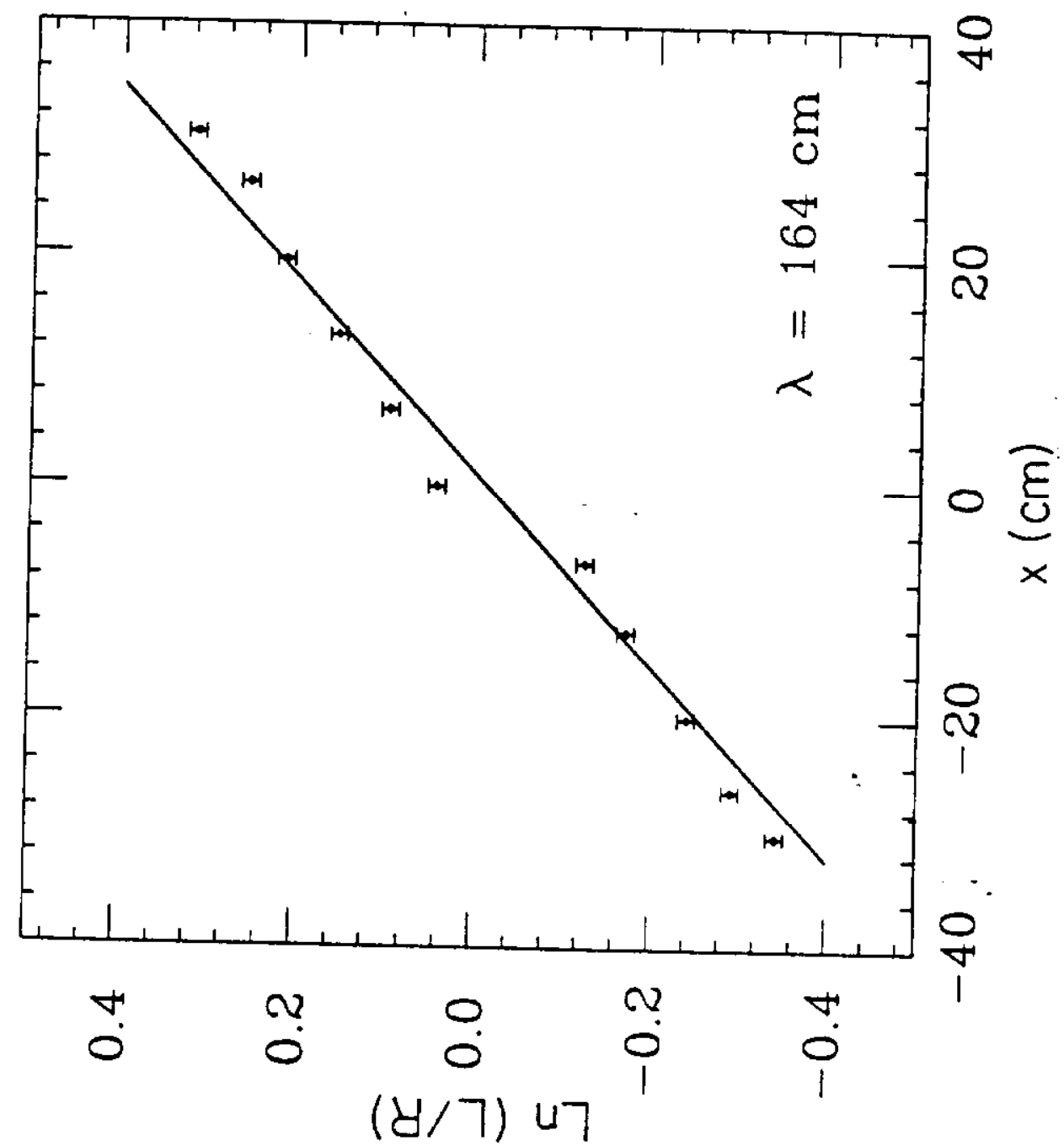




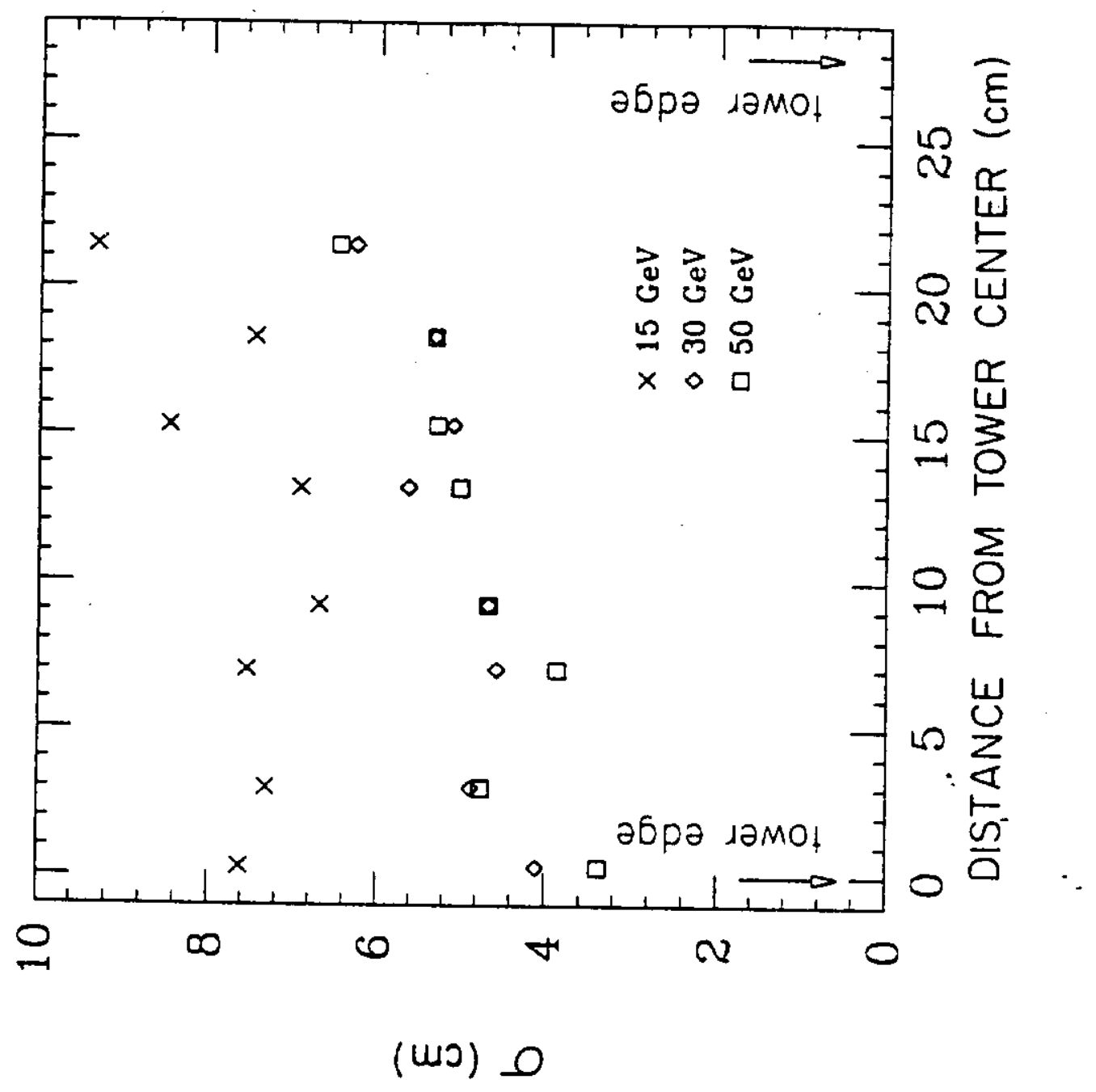

ส น음 


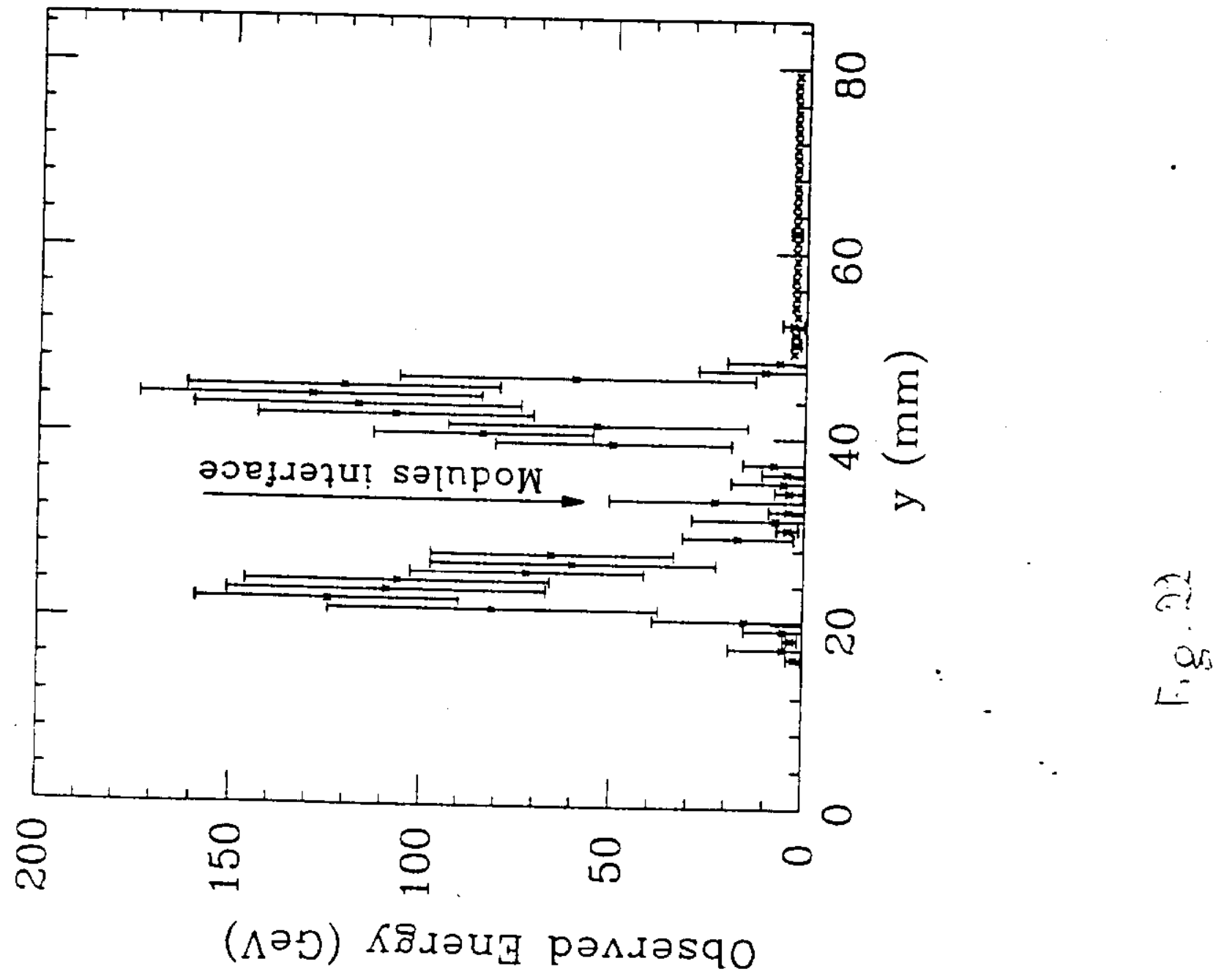


w

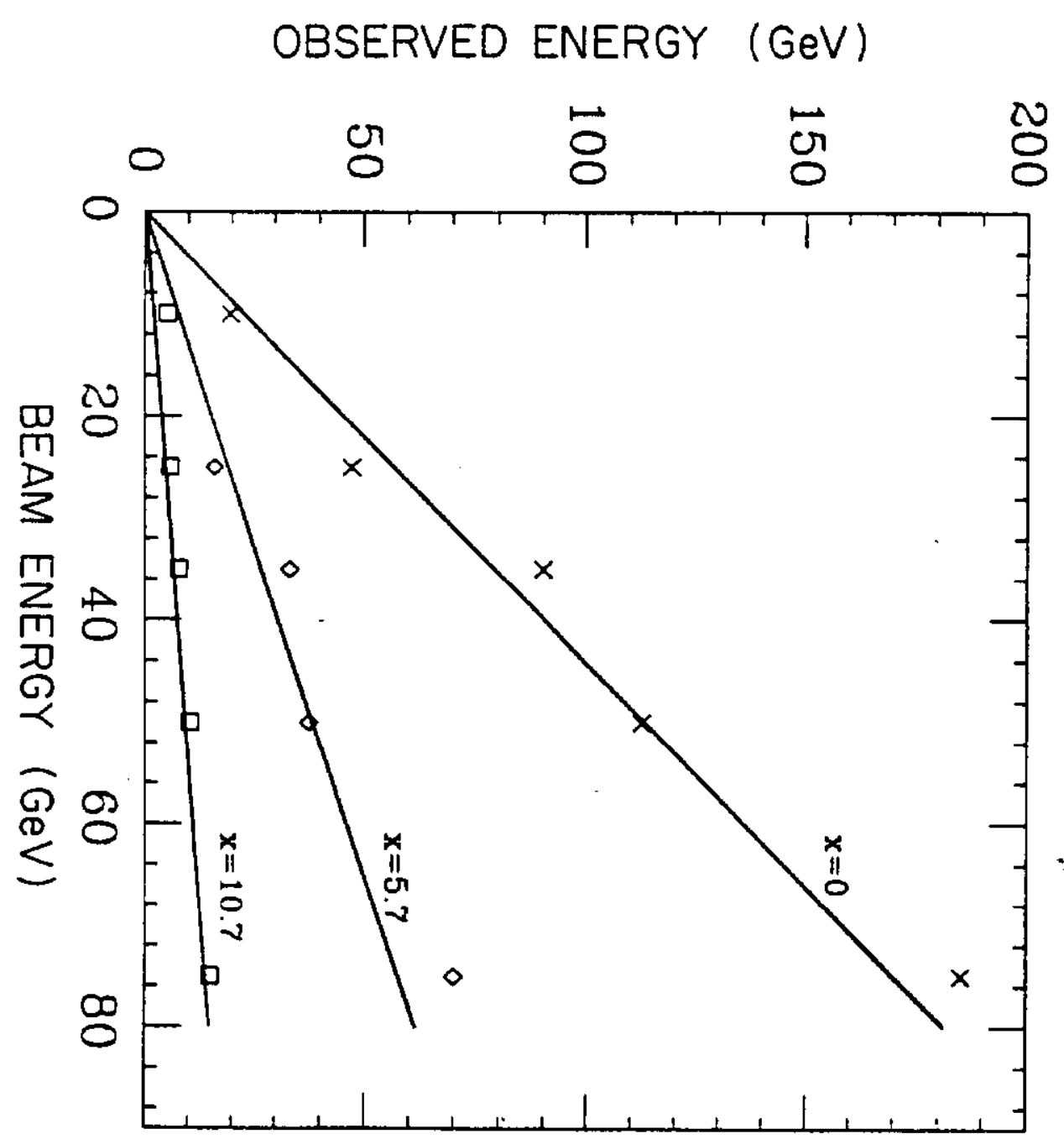




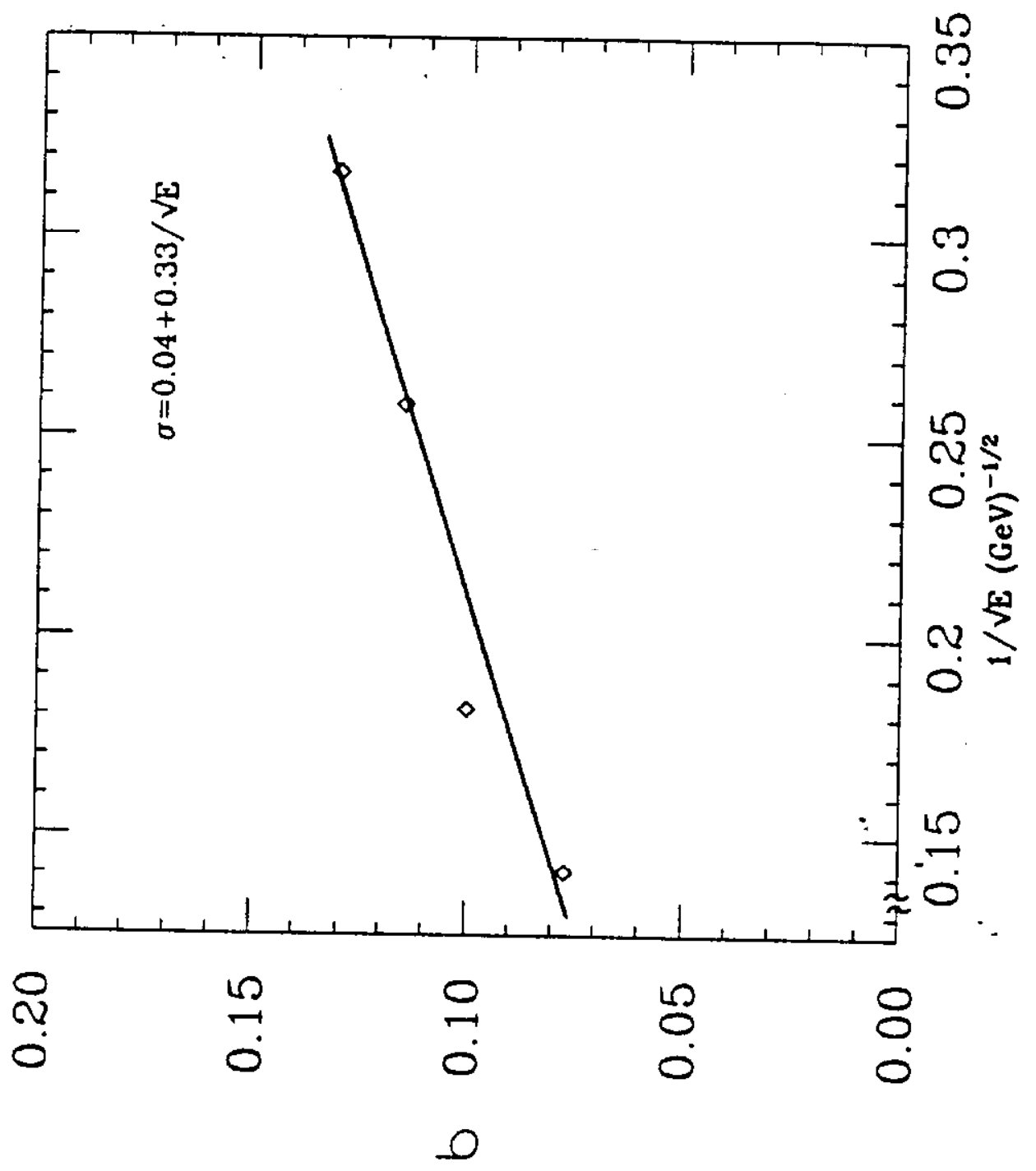

$\overrightarrow{8}$ 
$\frac{T}{0}$

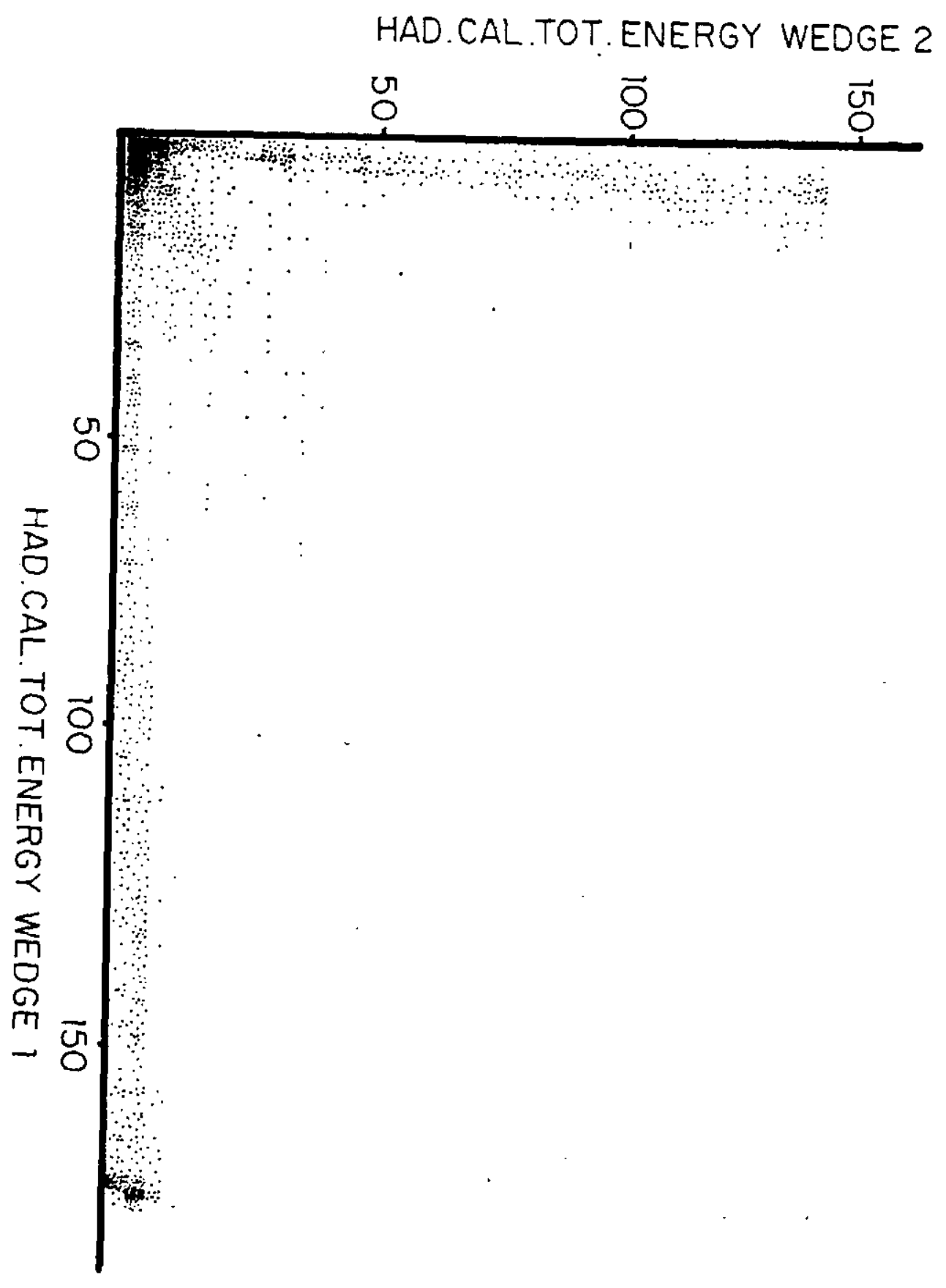


$\frac{\pi}{0}$

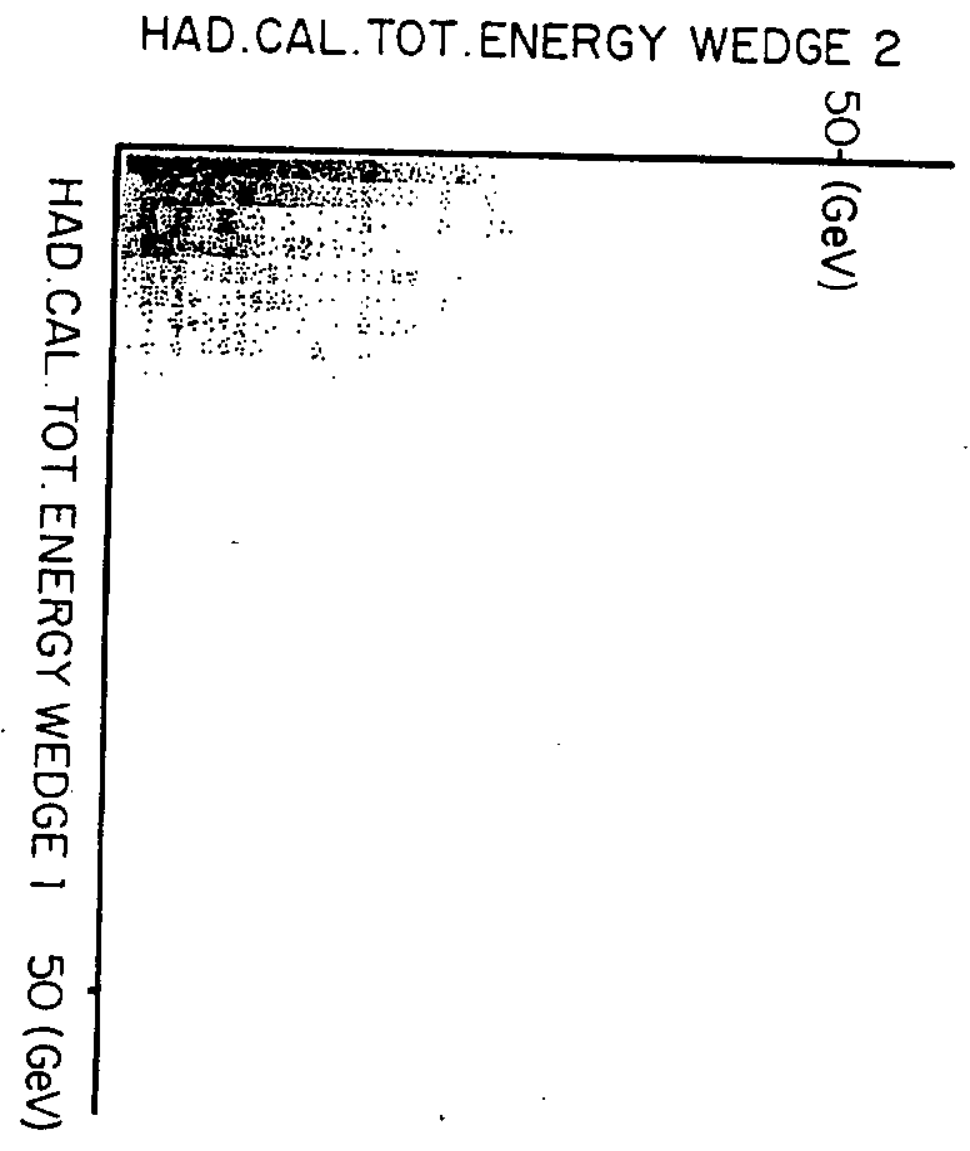


مَ

5

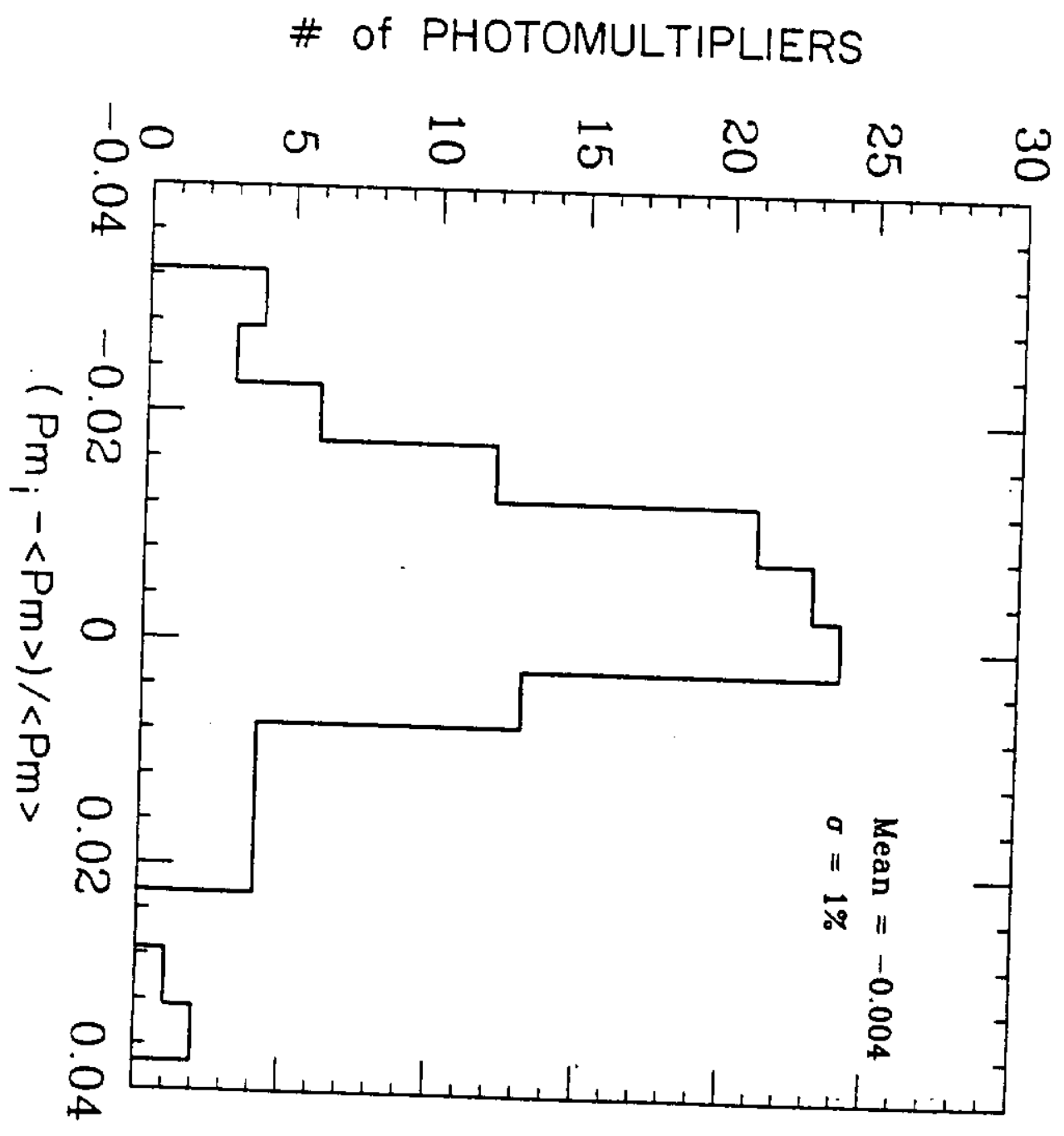




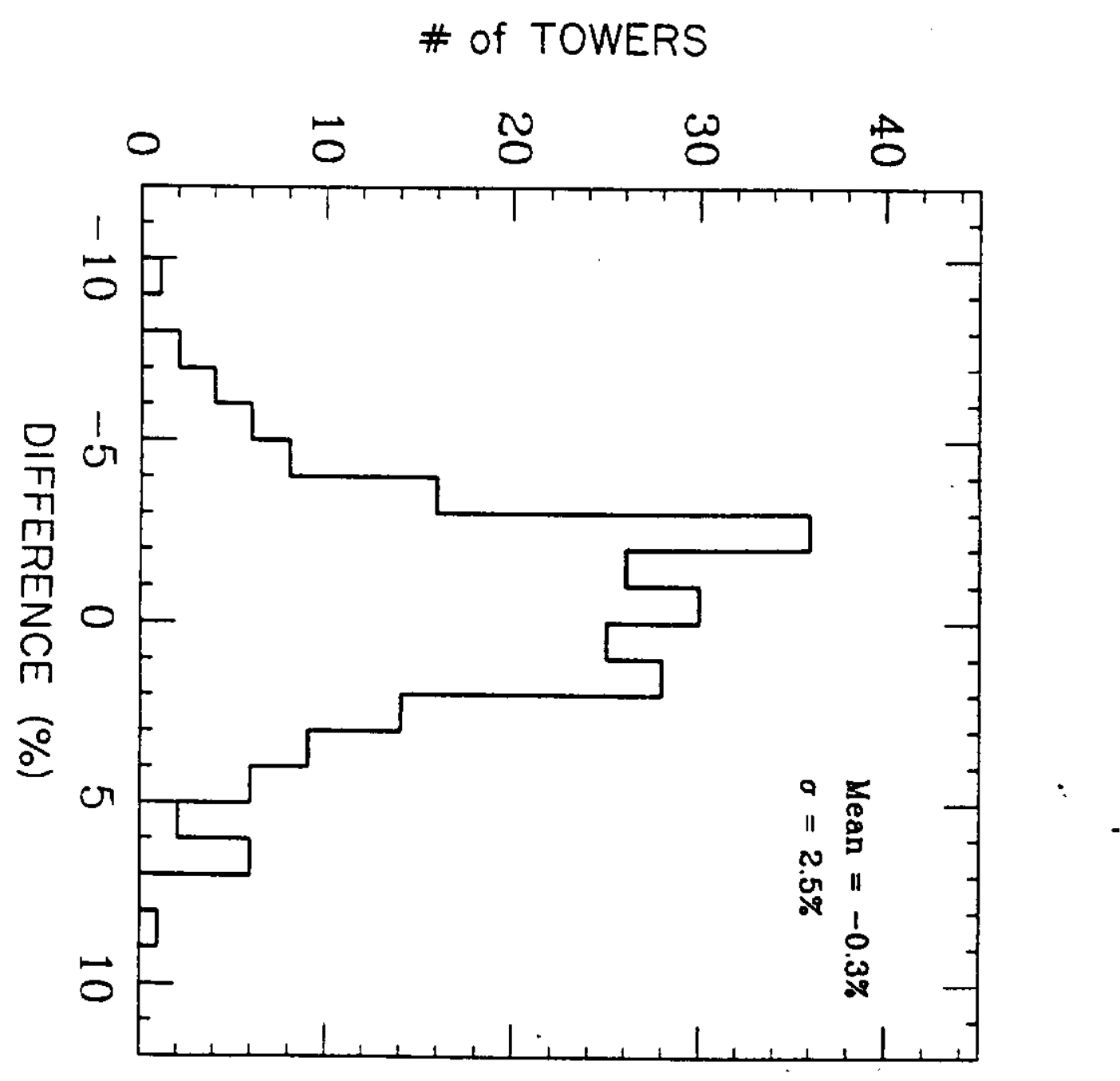


$\frac{\pi}{0}$

\# of PHOTOMULTIPLIERS

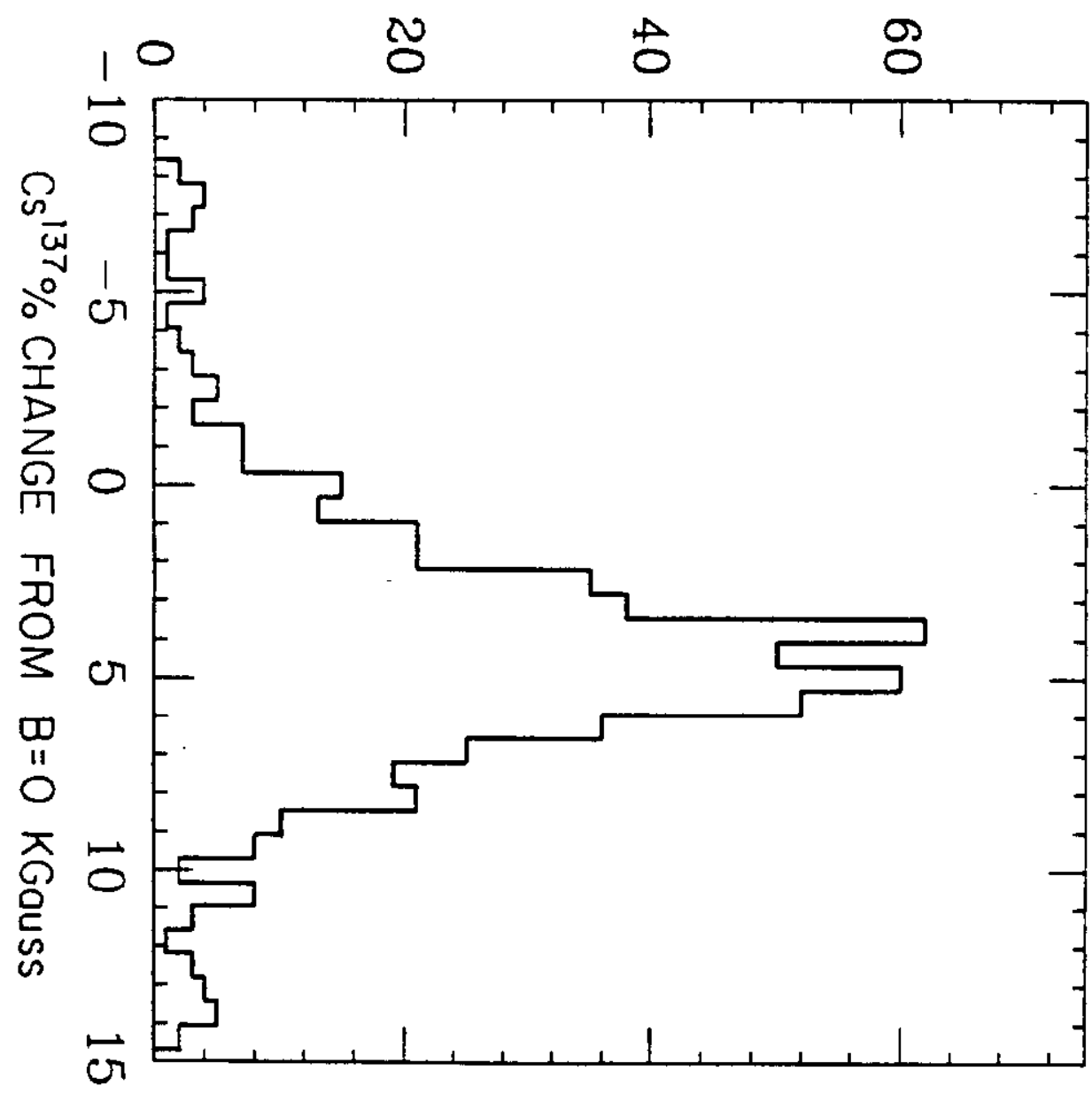


$\frac{\pi}{0}$

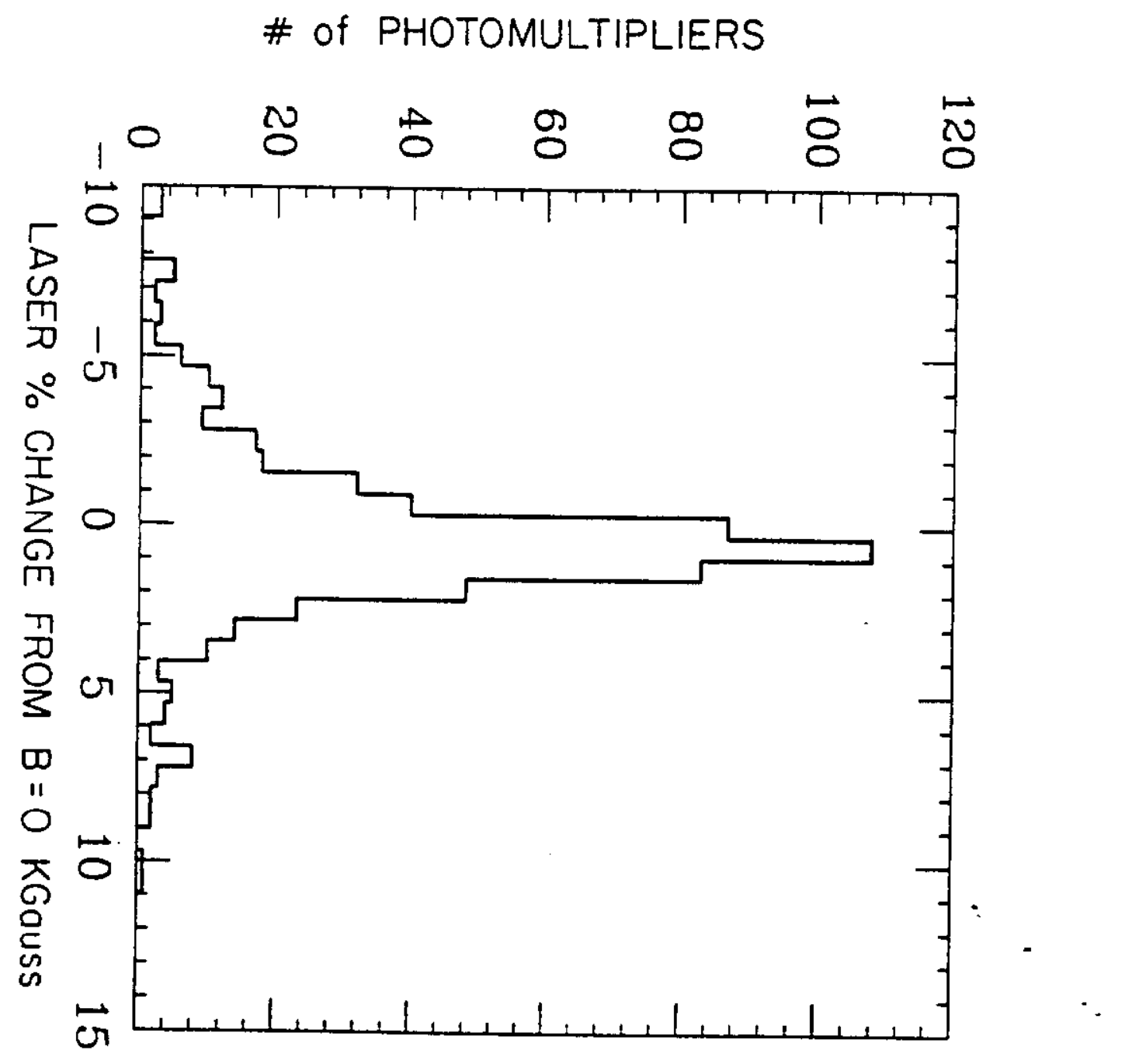


\# of PHOTOMULTIPLIERS
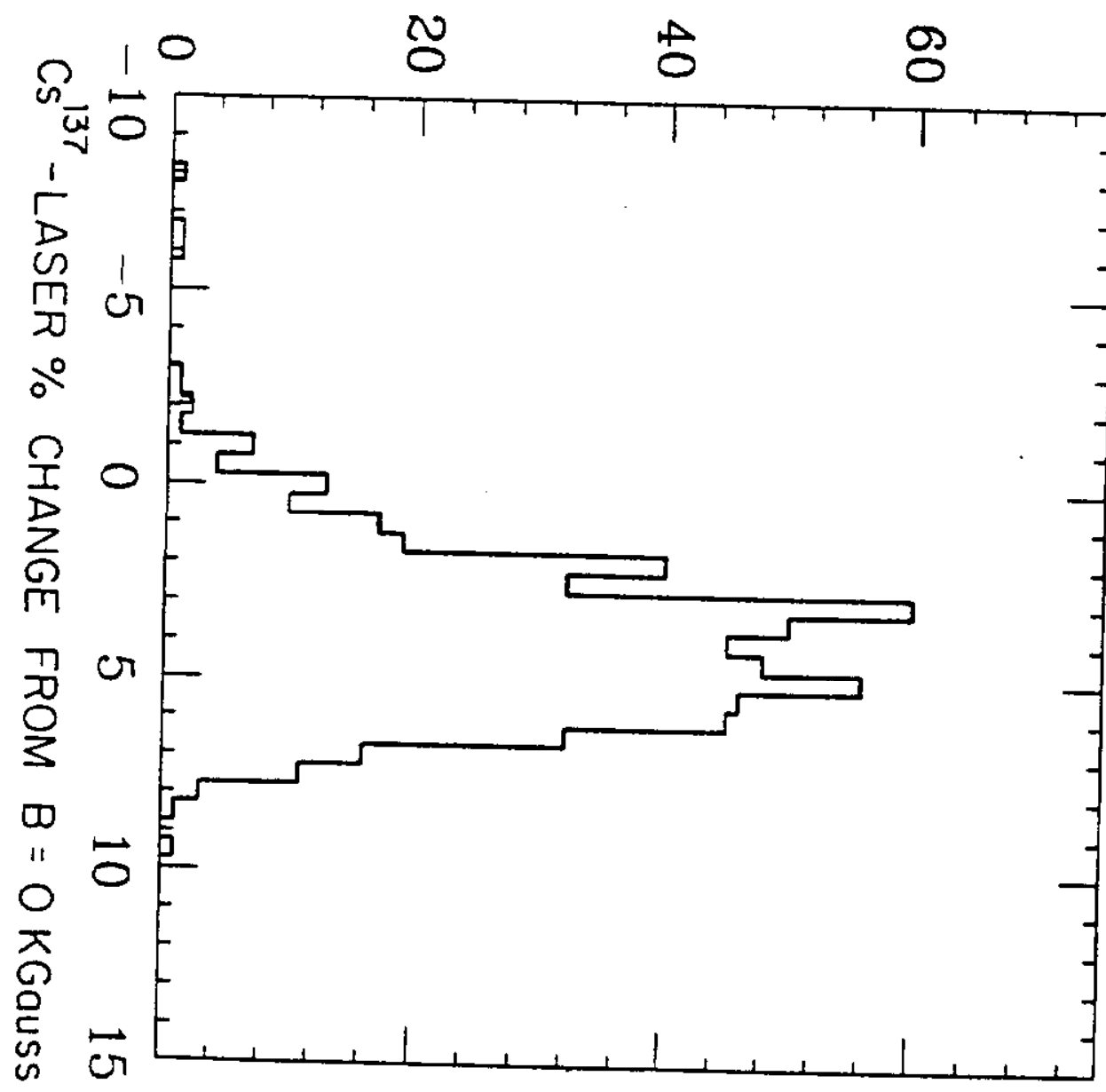


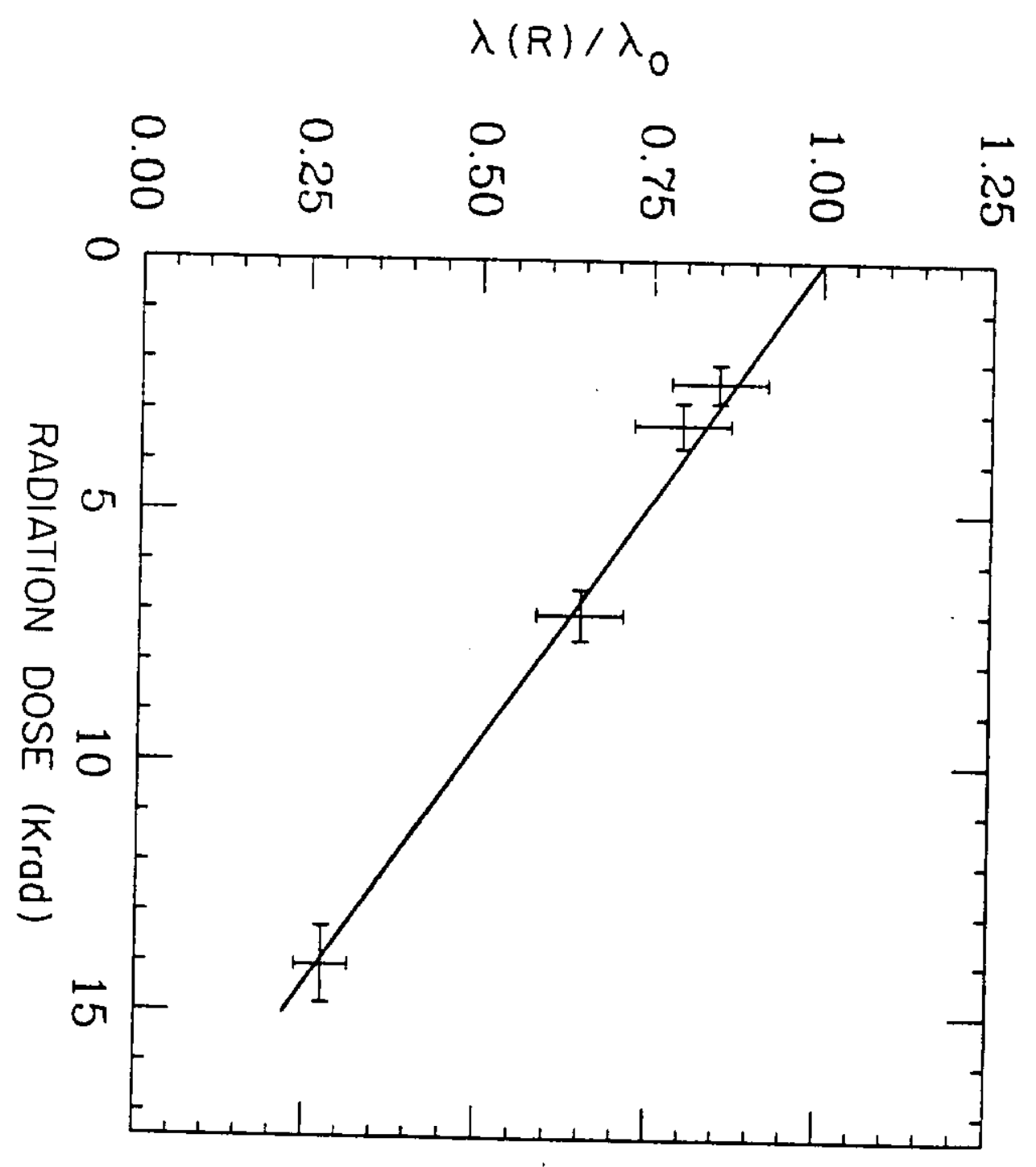

